\title{
Bimetallic Fe-Ag arrays with extraordinary nonlinear refraction and nonlinear Faraday rotation at telecommunication wavelength $(1550 \mathrm{~nm})$
}

\section{Krishna Prasad Koirala}

the University of Tennessee, Knoxville https://orcid.org/0000-0002-9137-917X

Hernando Garcia

Southern Illinois University in Edwardsville

Venkatanarayana Prasad Sandireddy

the University of Tennessee, Knoxville

Ramki Kalyanaraman

University of Tennessee at Knoxville https://orcid.org/0000-0002-5340-029X

Gerd Duscher ( $\nabla$ gduscher@utk.edu )

The University of Tennessee Knoxville

\section{Article}

Keywords: Bimetallic Fe-Ag arrays, nonlinear refraction, nonlinear Faraday rotation, telecommunication wavelength, magneto-optical materials and devices

Posted Date: September 28th, 2020

DOl: https://doi.org/10.21203/rs.3.rs-79014/v1

License: (c) (1) This work is licensed under a Creative Commons Attribution 4.0 International License.

Read Full License 


\title{
Bimetallic Fe-Ag arrays with extraordinary nonlinear refraction and nonlinear Faraday rotation at telecommunication wavelength $(1550 \mathrm{~nm})$
}

\author{
Krishna Prasad Koirala ${ }^{1}$, Hernando Garcia ${ }^{2}$, Venkatanarayana \\ Prasad Sandireddy ${ }^{3}$, Ramki Kalyanaraman ${ }^{3,4}$, Gerd Duscher ${ }^{4,5}$ \\ ${ }^{1}$ Department of Physics and Astronomy, University of Tennessee, Knoxville, TN, 37996, USA \\ ${ }^{2}$ Department of Physics, Southern Illinois University, Edwardsville, IL, 62026, USA \\ ${ }^{3}$ Department of Chemical and Biomolecular Engineering, \\ University of Tennessee, Knoxville, TN, 37996, USA \\ ${ }^{4}$ Department of Material Science and Engineering, \\ University of Tennessee, Knoxville, TN, 37996, USA and \\ ${ }^{5}$ Materials Science and Technology Division, Oak Ridge National Laboratory, Oak Ridge, 37830,USA \\ There is a pressing need to discover magneto-optical materials and devices with better perfor- \\ mance and lower cost that operate at telecommunication wavelengths. Here we report the discovery \\ of giant negative nonlinear refraction and nonlinear Faraday rotation at $1550 \mathrm{~nm}$ using an array of \\ bimetallic Fe-Ag nanopyramids. This system exhibited a very large third order nonlinear refractive \\ index $\left(n_{2}=-2.32 \mathrm{~cm}^{2} / \mathrm{GW}\right)$ and nonlinear figure of merit $(F=2.3)$. The same system also exhib- \\ ited an extraordinarily large magneto-optical susceptibility $\left(\chi_{i}^{4}=6.5 \times 10^{-12} \mathrm{esu}\right)$ and photoinduced \\ nonlinear Faraday rotation up to $2.5 \mathrm{radian} / \mu \mathrm{m}$ at a magnetic field of $0.5 \mathrm{~T}$. The nonlinear response \\ was dependent on the degree of overlap of the Fe nanopyramid on the Ag nanopyramid which influ- \\ ences the strength of plasmon induced dipoles on the Ag nanopyramid. This nanoscale system opens \\ up a rich new set of possibilities in utilizing magneto-plasmonic materials to miniaturize future mul- \\ tifunctional devices at telecommunication wavelengths.
}




\section{MAIN}

The wavelength-dependent optical and magneto-optical properties of metal nanostructures are an area of active research due to a variety of potential applications. One area of particular interest is their nonlinear optical (NLO) and nonlinear magneto-optical (NLMO) properties at or near the telecommunication wavelengths (such as between $1530 \mathrm{~nm}-1570 \mathrm{~nm}$ ), that result from utilizing suitably engineered materials design [1-3]. Large and ultrafast optical and magneto-optical nonlinearity are generally desired for optical communication functionalities like all-optical switching, optical isolation, pulse limiting, multiplexing, demultiplexing, high sensitive magnetometry and quantum information processing [4-13]. Silicon has been considered to be a promising material for all-optical logic at telecommunication wavelength due to its linear optical transparency and a large nonlinear refractive index of $n_{2}=4 \times 10^{-5} \mathrm{~cm}^{2} / \mathrm{GW}$. However, its ultimate performance is limited by a weak nonlinear figure of merit of $F=0.35$ defined by $F=\frac{n_{2}}{\beta \lambda}$, where $\beta$ is the nonlinear absorption coefficient and $\lambda$ is the wavelength of the incident light [14]. Graphene has also been regarded as a promising material due to the very high $n_{2}$ of $102 \mathrm{~cm}^{2} / \mathrm{GW}$ at the telecommunication wavelength. However, it is unlikely to address the current demand of high performance optical devices due to the challenges with making large area graphene for mass production as well as its zero band gap which results on broad-band optical absorption [15]. Many other 2D materials have been proposed for all-optical devices, including hexagonal Boron Nitride (h-BN), metal oxides, perovskites and metal dichalcogenides [16-23]. Despite exciting advances, outstanding challenge remain in the large-area-growth of low defect materials along with intrinsic materials limitations, such as a Faraday rotation limited by the cyclotron frequency of the system. The current state-of-the-art in the field of strong Faraday rotators at telecommunication wavelengths are based on linear response of bulk synthetic materials and solely driven by Yttrium Iron Garnet (YIG) [24]. Nonlinear Faraday properties have been generally investigated for cold atoms and atomic vapors at or near the laser wavelengths (energies) of their hyperfine transition energies regions, and for some dilute magnetic semiconductors like CdMnTe at or near their optical band gap energies, which are far from the telecommunication wavelength [12, 25, 26]. To the best of our knowledge, the nonlinear Faraday process has not been studied on the material systems at or near the telecommunication wavelengths. Partially motivated by these limitations, and the many unusual plasmonic and magneto-plasmonic properties of metallic nanostructures that have been discovered recently, there is continuing interest among the nonlinear optics and magneto-optics community to explore plasmonic nanostructures at the important $1550 \mathrm{~nm}[27-31]$.

One class of interesting metallic systems are bimetallic plasmonic nanostructures which show either improved materials stability and/or unusual optical and magneto-optical properties in terms of activity and 
stability arising from synergetic effects. In the past, metals like $\mathrm{Fe}, \mathrm{Co}, \mathrm{Ni}, \mathrm{Pt}, \mathrm{Pd}$ and $\mathrm{Al}$ have been combined with the noble metals $\mathrm{Ag}, \mathrm{Au}$ and $\mathrm{Cu}$ to enhance the plasmonic and magneto-plasmonic properties. For example, ferroplasmons have been discovered in Ag-Co bimetallic nanoparticles, in which strong surface plasmons have been observed in the Co side of the metallic system while preserving its ferromagnetism [32, 33]. Additionally, the Ag-Co and Ag-Fe system have also demonstrated reduced degradation rate of plasmonic signals due to oxidation of silver thus leading to very stable plasmonic behavior over long periods of time [33, 34]. $\mathrm{Fe}_{2} \mathrm{O}_{3}$-Au core shell nanoparticles have been reported to have a high Faraday rotation at resonant frequency ascribed to spectral overlap of magneto-optical transition to plasmonic resonance [35].

In this article, we report the discovery of giant third order nonlinear refractive index and photo-induced nonlinear Faraday rotation at $1550 \mathrm{~nm}$ from a hexagonal array of partially overlapped $\mathrm{Fe}-\mathrm{Ag}$ truncated nanopyramids. This nanopyramidal system exhibited a very large third order nonlinear refractive index $\left(n_{2}\right.$ $\left.=-2.32 \mathrm{~cm}^{2} / \mathrm{GW}\right)$ and nonlinear figure of merit $(F=2.3)$. The same system also exhibited a photoinduced nonlinear Faraday rotation of magnitude of $2.5 \mathrm{radian} / \mu \mathrm{m}$ at a magnetic field of $0.5 \mathrm{~T}$. We found that these effects are strongly dependent on the plasmon induced dipole strength from Ag nanopyramids across the Fe nanopyramids and which in turn depends on the overlapping extent of Fe nanopyramids on $\mathrm{Ag}$ nanopyramids.

\section{RESULTS}

\section{Nonlinear optical properties}

We studied nonlinear optical properties of nanopyramidal systems viz. pure $\mathrm{Ag}$, pure $\mathrm{Fe}$ and $\mathrm{Fe}-\mathrm{Ag}$ (with $30 \%$ and $90 \%$ overlap) at $1550 \mathrm{~nm}$ whose surface plasmons induced resonant absorption was at $750 \mathrm{~nm}$ (except for pure Fe pyramidal system). The third order optical nonlinearities were studied by f-scan methods, particularly transmission f-scan and reflection f-scan, which are modifications of the conventional z-scan method [36-38]. These methods allow us to measure the nonlinear index of refraction $\left(\mathrm{n}_{2}\right)$ in reflection and the nonlinear absorption or two photon absorption coefficient $(\beta)$ in transmission modes. The electric field (E) dependent polarization $(\mathbf{P})$ of a nonlinear system can be expressed as

$$
\mathbf{P}=\chi_{E}^{(1)} \mathbf{E}+\mathbf{P}_{\mathbf{N L}}=\chi_{E}^{(1)} \mathbf{E}+\chi_{E E}^{(2)} \mathbf{E E}+\chi_{E E E}^{(3)} \mathbf{E E E}+\chi_{E E E E}^{(4)} \mathbf{E E E E}+\ldots
$$

where, $\chi^{(i)}(i=1,2,3 \ldots)$ is the $i^{\text {th }}$ order nonlinear susceptibility of the nonlinear system. The real part of the

$\chi_{E E E}^{(3)}$ is related to $n_{2}$ while imaginary part is related to $\beta$. A schematic of experimental set up to calculate the value of $n_{2}$ and $\beta$ is shown in Fig. 1 (a). First, for the open aperture configuration, we used a 1550 
$\mathrm{nm}$ fiber laser with a pulse duration of $64 \mathrm{fs}$, and an average power of $20 \mathrm{~mW}$ which was incident on an electrically focused tunable lens (EFTL). The samples were placed at distance $\mathrm{f}_{s}$ from the EFTL. The output of the lens impinged in the sample and the intensity in the sample was controlled by changing the focal length of the EFTL with applied current. The transmitted laser beam was detected with a Ge-photodetector, and the normalized transmittance was used to calculate the value of $\beta$ using the relation as,

$$
T(f)=\frac{1}{B(f)} \int \ln \left\{1+B(f) \operatorname{sech}^{2}(\rho)\right\} d \rho, \rho \rightarrow 0, \infty
$$

with

$$
B(f)=\beta(1-R) I_{o}(f) L_{e f f}
$$

Here, $L_{e f f}$ is the effective sample thickness given by $L_{e f f}=\frac{\left(1-e^{-\alpha L}\right)}{\alpha}, L$ being sample thickness and $\alpha$ being linear absorption coefficient. Also, $R$ is reflection coefficient and $I_{o}$ is peak intensity of the beam which is function of focal length. Also, $\rho$ is expressed as $\rho=\frac{2 \ln (1+\sqrt{\tau})}{\tau}$ with $\tau$ being full width at half maximum pulse duration.

Second, the samples were tilted at an angle of $\theta$ with respect to the laser beam direction and the light reflected by the sample surface was analyzed to measure the $n_{2}$. The normalized reflected beam intensity can be expressed as

$$
R_{N}(f, \theta)=1+\operatorname{Re}\left[\frac{2 n_{o}^{3} \cos \theta-4 n_{o}^{2} \sin ^{2} \theta \cos \theta}{n_{o}^{4} \cos ^{2} \theta-n_{o}^{2}+\sin ^{2} \theta}\right]\left[\frac{\left(n_{2}+i k_{2}\right) I_{o}}{\sqrt{n_{o}^{2}-\sin ^{2} \theta}}\right]\left\{\frac{1}{1+\left(\frac{f_{s}-f}{Z_{0}(f)}\right)^{2}}\right\}
$$

Here, $f, Z_{0}(f), \theta, n_{o}$ and $k_{2}$ are the focal length of EFTL, Rayleigh range, angle between normal to the incident beam and sample surface, linear refractive index and extinction coefficient respectively.

Fig. 1(b) and (c) show results for transmission and reflection f-scans for the pure Fe, pure Ag and FeAg nanopyramidal systems. Two immediate features are evident from Fig. 1 b) and (c). First, the Fe-Ag system exhibited significantly enhanced nonlinear absorption and refraction as compared to the pure $\mathrm{Ag}$, which in turn was much higher than pure Fe. Second, the Fe-Ag system showed the opposite sign for the nonlinear refraction as compared to pure Ag. A very arge value for the nonlinear refraction of $n_{2}^{F e-A g}=$ $-2.32 \mathrm{~cm}^{2} / \mathrm{GW}$ and nonlinear absorption coefficient of $\beta^{F e-A g}=6.5 \times 10^{3} \mathrm{~cm} / \mathrm{GW}$ were obtained for the Fe-Ag system with $30 \%$ overlap. For the Fe-Ag system with $90 \%$ overlap, $n_{2}^{F e-A g}=-1.02 \mathrm{~cm}^{2} / \mathrm{GW}$ and $\beta^{F e-A g}=9.5 \times 10^{3} \mathrm{~cm} / \mathrm{GW}$ were obtained. Whereas, for the pure Ag system, the nonlinear refraction of $n_{2}^{A g}$ $=0.19 \mathrm{~cm}^{2} / \mathrm{GW}$ and nonlinear absorption coefficient of $\beta^{A g}=6.7 \times 10^{2} \mathrm{~cm} / \mathrm{GW}$ were obtained. For the pure Fe system the nonlinear response was negligible. The $n_{2}^{F e-A g}$ value is 3 to 4 orders of magnitude larger than those typically reported in the literature for metallic nanostructures, which range in the order of $10^{-4}$ $\mathrm{cm}^{2} / \mathrm{GW}$ to $10^{-1} \mathrm{~cm}^{2} / \mathrm{GW}$ [2, 39-41]. 


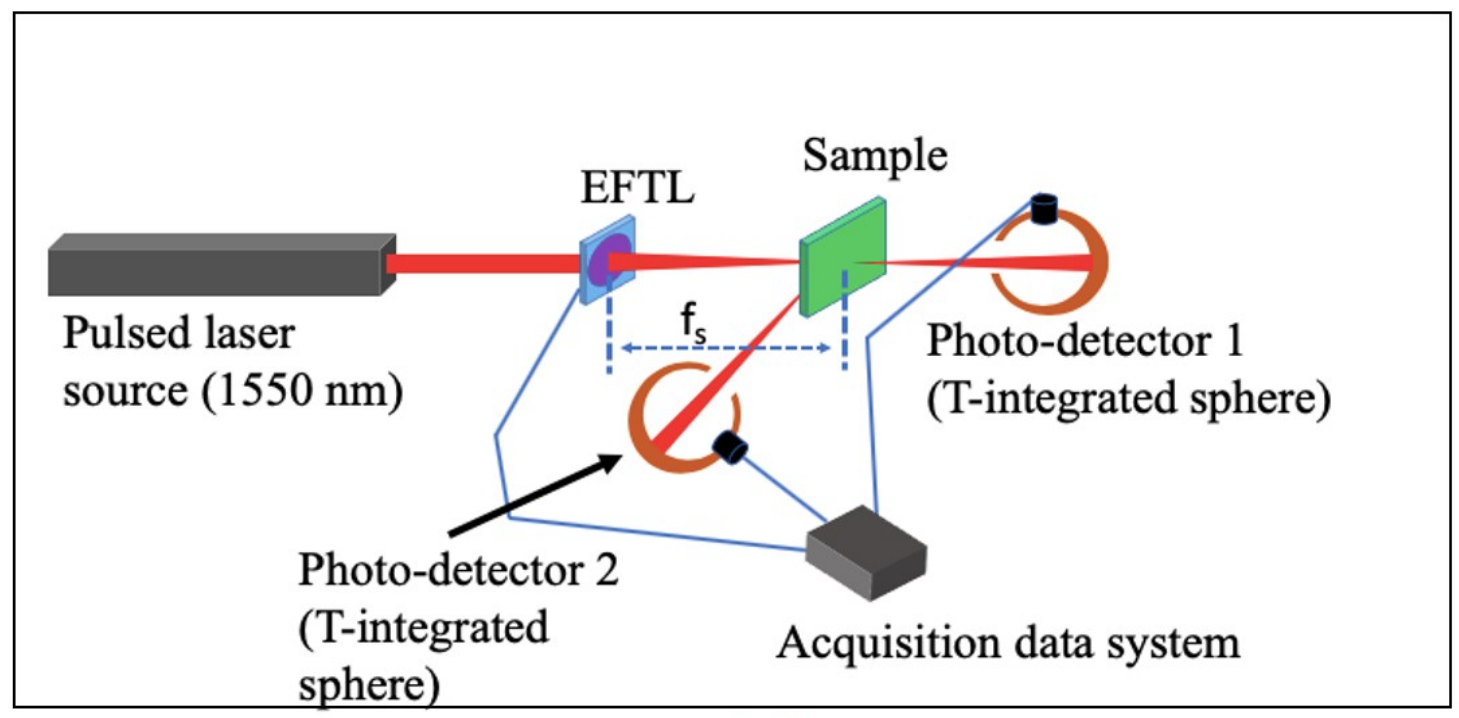

(a)

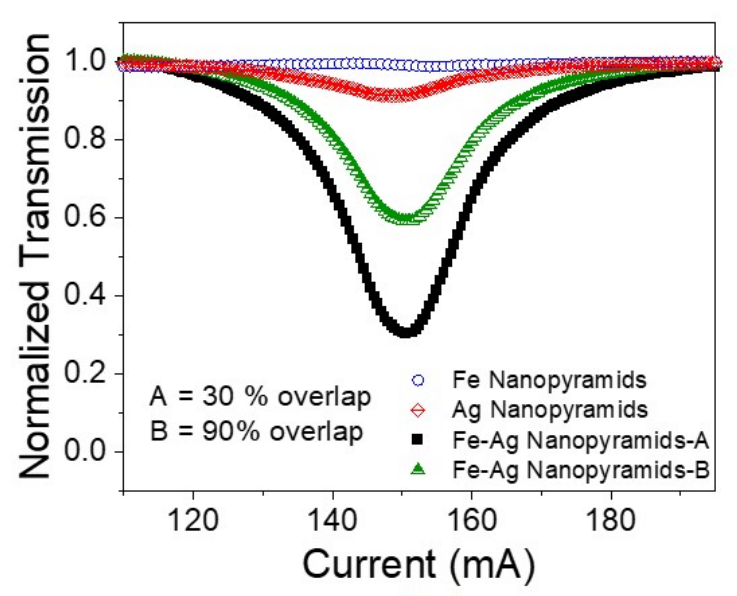

(b)

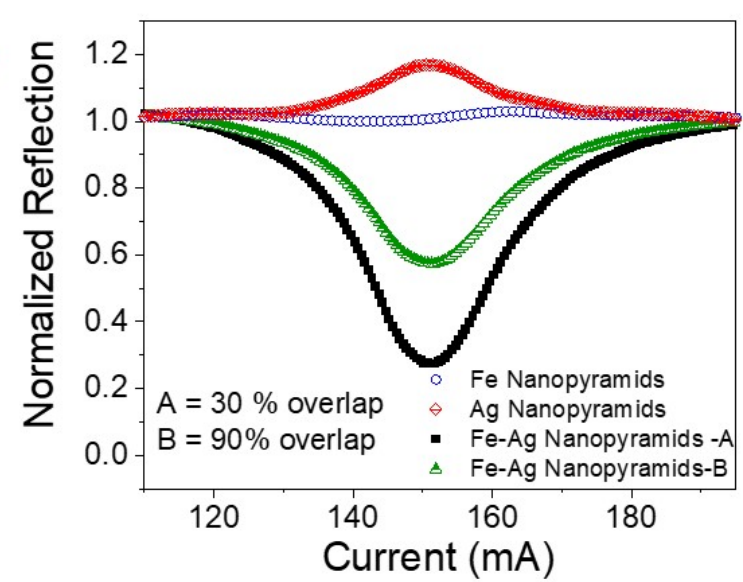

(c)

Figure 1. (a) Schematic diagram for experimental setup of f-scan method (b) normalized transmission and (c) normalized reflection of $\mathrm{Fe}, \mathrm{Ag}$ and $\mathrm{Fe}-\mathrm{Ag}$ nanopyramidal arrays.

\section{Plasmonics and microstructure}

In order to understand the origin of giant optical nonlinearity observed in the Fe-Ag nanopyramidal system, we investigated the plasmon induced linear optical behavior and electron energy-loss behavior. The plasmon induced linear optical absorption properties of the nanopyramidal system [pure Ag, pure Fe, $\mathrm{Fe}-\mathrm{Ag}$ (30\% and $90 \%$ overlap)] were measured using normally incident broadband light. We saw that the pure Ag has a sharper dipole resonance curve followed by Fe-Ag ( $\sim 30 \%$ overlap) and Fe-Ag ( $90 \%$ overlap) at 750 
$\mathrm{nm}$ (near the energy of two photons at $1550 \mathrm{~nm}$ ) as shown in Fig. 2(a). The dipole resonance peak at $750 \mathrm{~nm}$ was achieved in each pyramidal system by controlling the thickness of the Ag pyramids which was $\sim 17 \mathrm{~nm}$ while the height of the Fe pyramids in each case was $\sim 9 \mathrm{~nm}$. The representative SEM image of pure Ag and $30 \%$ overlapped Fe-Ag nanopyramidal system is shown in Fig. 2(b) and (c). The pure Fe nanopyramidal system didn't show any significant absorption features in the wavelength region studied here. Moreover, we didn't see any linear absorption feature in the $1550 \mathrm{~nm}$ in all nanopyramidal systems as evident from the inset of Fig. 2(a). The enhancement in optical nonlinearity observed in the pure Ag or the Fe-Ag system compared to that of the pure Fe system was attributed to matching of the two photon resonance conditions. The plasmonic systems ( $\mathrm{Ag}$ and Fe-Ag) had plasmon induced resonance peak wavelengths at $750 \mathrm{~nm}$ which is near the fundamental frequency for two photons at $1550 \mathrm{~nm}$. However, the giant enhancement in the Fe-Ag system over the Ag system was completely unexpected since the plasmon induced dipole resonance is more damped in the Fe-Ag system compared to the Ag system, as evident from Fig. 2(a). Nevertheless, this analysis firmly established that the matching of two photon resonance conditions partially contributed to the observed optical nonlinearity, as pure $\mathrm{Ag}$, and Fe-Ag nanopyramidal system had significantly higher optical nonlinearity than pure Fe.

One of the reasons for the very large nonlinear optical responses from Fe-Ag nanopyramidal array could be a synergistic effect between Fe metal and Ag metal nanopyramids. To explore the system further, we investigated the electron energy-loss spectra from those nanostructures as shown in Fig. 3(a), (b) an (c). The dipole induced electron energy-loss peaks were observed at $1.60 \pm 0.02 \mathrm{eV}$ in each case, slightly shifted from the observed optical absorbance at $1.65 \pm 0.01 \mathrm{eV}(750 \mathrm{~nm})$. This difference in observed electron energy-loss and optical absorbance peak comes from their observed part of the dielectric function. In case of EELS, we observe $\operatorname{Im}\left(\frac{\varepsilon-1}{\varepsilon+1}\right)$, while in optical absorption we observe $\operatorname{Im}(\varepsilon), \varepsilon$ being the complex dielectric function of the system [42]. To analyze the energy-loss behavior from the corner of Ag nanopyramids, the features in electron energy-loss peak from Ag corners [marked as A, B, C in Fig. 3] of each Ag, Fe-Ag (30 \% overlap) and Fe-Ag (90\% overlap) were quantified in terms of peak intensity and area [in parts per million $(\mathrm{ppm})]$. We found that the peak intensity of pure Ag nanopyramids were higher than that of Fe-Ag, and the Fe-Ag (30\% overlap) had higher peak intensity than Fe-Ag (90\% overlap). Further, peak intensity and peak area of $\mathrm{Ag}$ corners (B and $\mathrm{C}$ ) adjacent to the Fe overlap were smaller than free corner (A). Moreover, $30 \%$ overlapped Fe-Ag nanopyramids had significantly larger intensity and area (at B and C corners) than $90 \%$ overlapped Fe-Ag nanopyramids as summarized in Table $[$.

What was apparant from the optical and EELS analysis of nanopyramidal system was that the surface plasmon dipoles are stronger for the $30 \%$ overlapped as compared to the $90 \%$ overlapped case. The plas- 


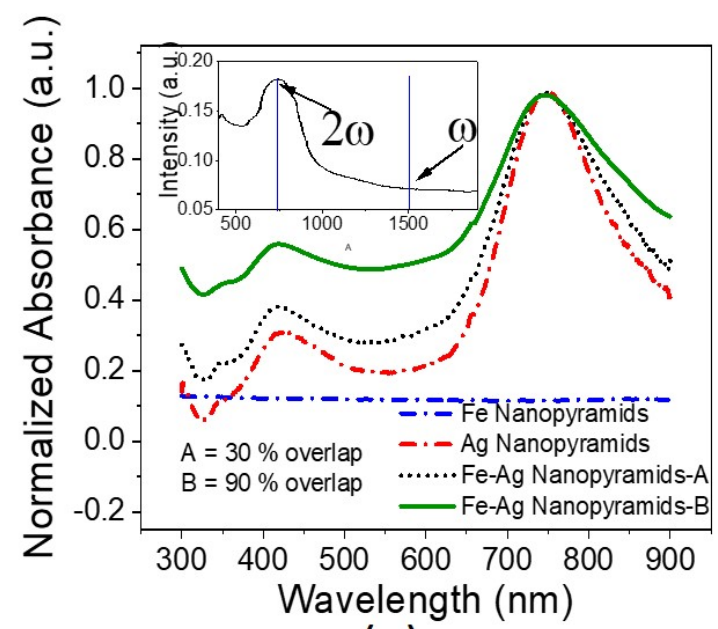

(a)

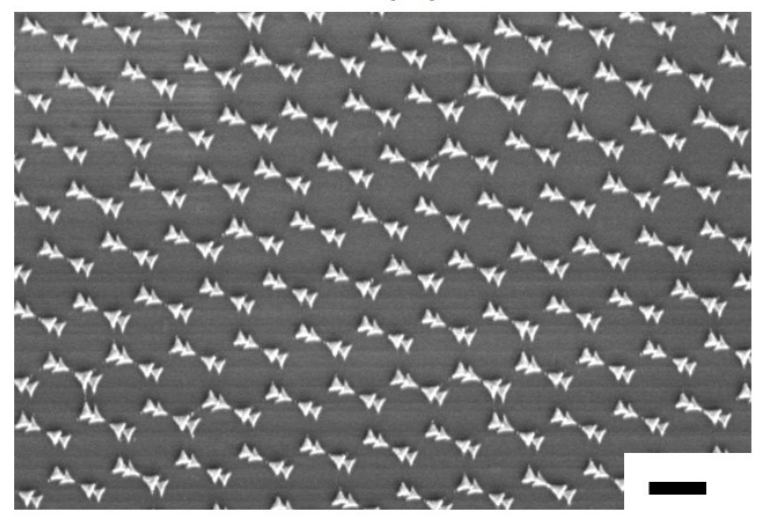

(c)

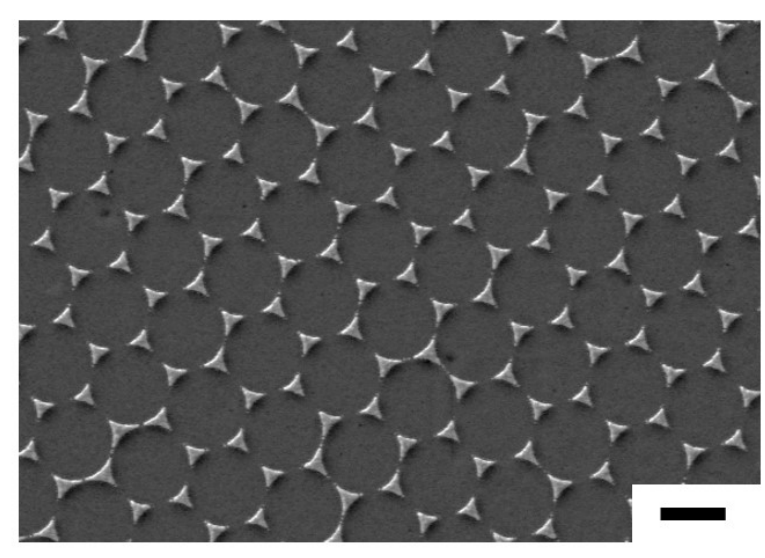

(b)

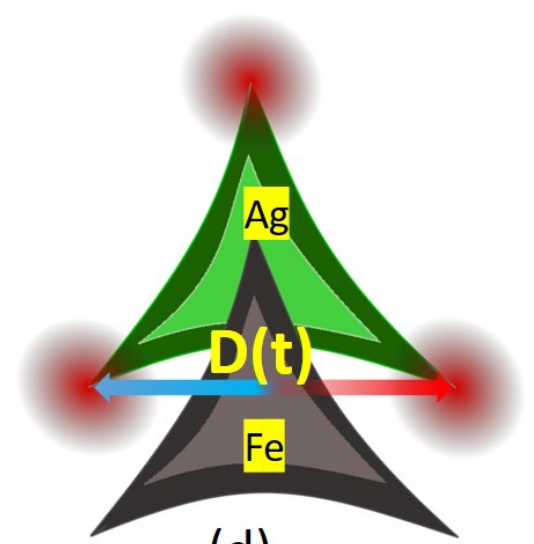

(d)

Figure 2. (a) Normalized plasmon induced linear absorption spectra of $\mathrm{Fe}, \mathrm{Ag}$ and $\mathrm{Fe}-\mathrm{Ag}$ (30 \% and $90 \%$ overlap) nanopyramidal systems. Inset shows the linear absorption of the Fe-Ag system from 400 to $2000 \mathrm{~nm}$. (b) A representative scanning electron micrograph (SEM) image of pure Ag and (c) Fe-Ag (30 \% overlapped system) (the scale bar is $500 \mathrm{~nm}$ ) (d)The schematic of oscillating displacement field $\mathrm{D}(\mathrm{t})$ in the Fe pyramid induced by surface plasmon dipoles of a Ag nanopyramid

mon induced oscillating dipole causes an induced electric displacement field across the Fe as in Fig. 2/d). The magnitude of displacement field is proportional to the plasmonic dipole strength. The displacement field developed across the ferromagnetic medium could control the magnetization of ferromagnetic material through magneto-electric effect. In other words, the dipole induced local field act on the electrons of the ferromagnetic material which produces magneto-electric (M-E) effect with moment $\mathbf{T} \sim \mathbf{P} \times \mathbf{M}$, where $\mathbf{P}$ 

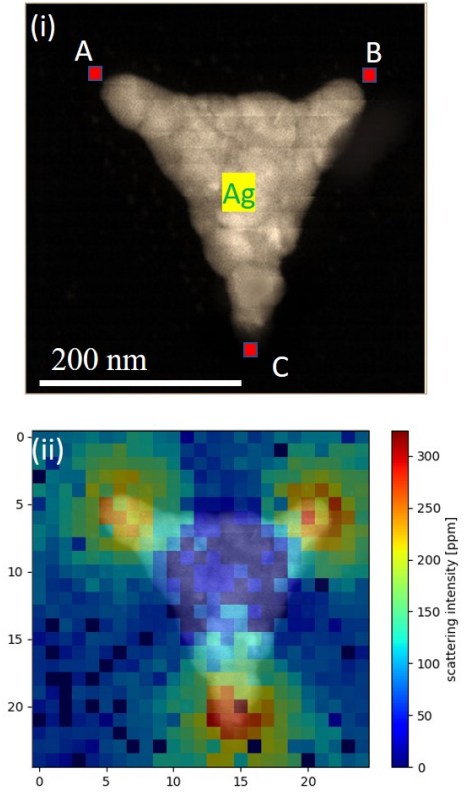

(a)
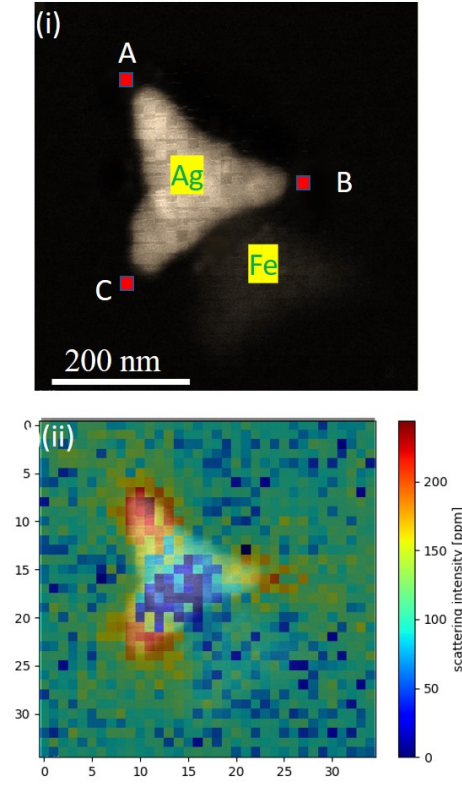

(b)
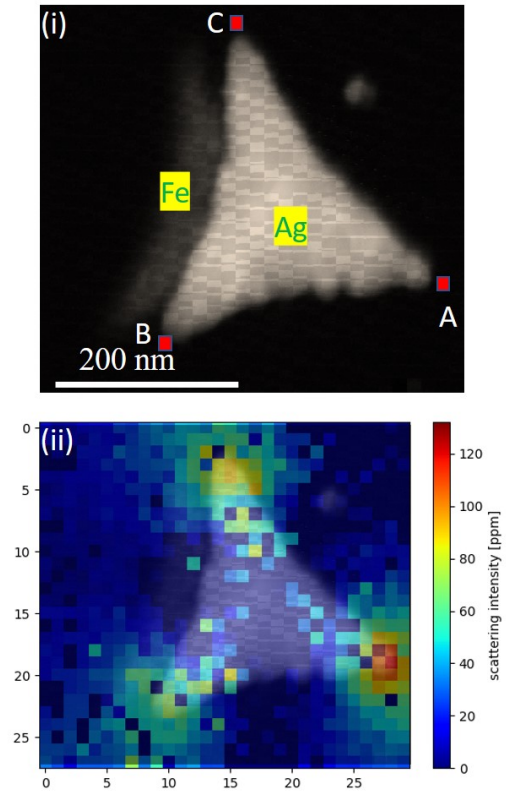

(c)

Figure 3. (i) High-angle annular dark-field images and (ii) EELS intensity mapping of (a) pure Ag, (b) Fe-Ag (30 \% overlapped), (c) Fe-Ag (90\% overlapped) nano pyramids at peak value of $1.60 \pm 0.02 \mathrm{eV}$.

\begin{tabular}{|c|c|c|c|c|c|c|c|c|c|}
\hline Peak at $1.60 \pm 0.02 \mathrm{eV}$ & \multicolumn{3}{|c|}{ Pure Ag } & \multicolumn{3}{c|}{ Fe-Ag (30\% overlap) } & \multicolumn{2}{|c|}{ Fe-Ag (90\% overlap) } \\
\hline \hline & A & B & C & A & B & C & A & B & C \\
\hline Peak intensity (ppm) & 232.2 & 235.5 & 240.0 & 180.2 & 165.4 & 168.2 & 120.2 & 86.9 & 90.5 \\
\hline Area (ppm) & 95.6 & 97.8 & 102.7 & 181.9 & 166.6 & 169.1 & 74.7 & 66.5 & 67.0 \\
\hline
\end{tabular}

Table I. The measurement average EELS peak intensity and average EELS peak area from Ag and Fe-Ag nanopyramids as marked regions A, B, C in Fig. 3 (ppm stands for part per million)

and $\mathbf{M}$ are plasmon dipole induced electric polarization and magnetization of ferro-electrons in Fe nanopyramids. This moment is expected to control the third order optical nonlinearity in nanomaterials [43, 44]. So, the plasmon induced effect in ferromagnetic material like M-E effect could be an important factor, apart from matching of the two photon resonance conditions, for enhancement of the nonlinear optical behavior in Fe-Ag nanopyramidal system.

\section{Nonlinear Faraday rotation}

Given the above hypothesis, we explored the possibility of external magnetic effects on the nonlinear behavior of this system by employing both the f-scan and polarization rotation measurements simultaneously, 
as suggested by Frey et al [45]. The Fe-Ag system, which showed the large value of $n_{2}$ (i.e. $30 \%$ overlap $\mathrm{Fe}-\mathrm{Ag}$ ), the nonlinear Faraday rotation at $1550 \mathrm{~nm}$ was studied. In the presence of external static magnetic field, the polarization of the system can be expressed as

$$
\mathbf{P}=\chi_{E}^{(1)} \mathbf{E}+\mathbf{P}_{\mathbf{N L}}^{H}=\chi_{E}^{(1)} \mathbf{E}+\chi_{E H}^{(2)} \mathbf{E H}+\chi_{E E E}^{(3)} \mathbf{E E E}+\chi_{E E E H}^{(4)} \mathbf{E E E H}+\ldots
$$

Here, $\chi_{E H}^{(2)}$ is the second-order nonlinear susceptibility which is related to linear Faraday rotation/magnetic circular dichroism (MCD) while $\chi_{E E E H}^{(4)}$ is the fourth-order nonlinear susceptibility which describes nonlinear Faraday (polarization) rotation.

The schematic diagram for the experimental set up to measure nonlinear Faraday rotation is shown in Fig. 4(a). The laser with linear polarized light was focused on the sample by the EFTL and a regular f-scan was taken as a function of the applied magnetic field in the Faraday configuration where the static magnetic field varied from 0 up to $0.5 \mathrm{~T}$ in steps of $0.1 \mathrm{~T}$. We recorded the transmission of the laser beam as a function of current in the EFTL at some fixed value of the magnetic field, after the beam was passed through a Thomson polarizer oriented at a $45^{\circ}$ angle with respect to the incoming polarization when there was no magnetic field applied. The transmitted intensity of the beam can be expressed as

$$
I(f)=\frac{I_{o}\left(f+f_{s}\right)}{1+\beta I_{o}\left(f+f_{s}\right) L_{e f f}}
$$

and the polarization angle of rotation $(\Delta \Theta)$ can be expressed as

$$
\Delta \Theta=V H L_{e f f}-\frac{\chi_{i}^{4}}{2 \chi_{i}^{3}} H \ln \left\{1+\beta I_{o}\left(f+f_{s}\right) L_{e f f}\right\}
$$

Where, $H$ is the applied magnetic field and $V$ is the Verdet constant, and $\chi_{i}^{4}$ and $\chi_{i}^{3}$ are the imaginary components of the fourth-order nonlinear and third-order nonlinear susceptibilities. The first term in equation 7 corresponds to the linear Faraday effect, while the second term is the photo-induced contribution to the nonlinear rotation. The transmittance of the beam passing through the analyzer can be calculated using Malus's law in the small angle approximation and optimizing for sensitivity as,

$$
T(f)=\left\{1-\beta I_{o}\left(f+f_{s}\right) L_{e f f}\right\}\left[1+V H L_{e f f}-\frac{\chi_{i}^{4}}{2 \chi_{i}^{3}} H \ln \left\{1+\beta I_{o}\left(f+f_{s}\right) L_{e f f}\right\}\right]
$$

At low intensity, we observed that all the scans showed the same value indicating that the linear Faraday rotation is negligible for the sample at $1550 \mathrm{~nm}$ (zero offset in the wings as a function of the B-field). This linear rotation behavior is expected due to the off-resonance condition (away from the plasmon resonance energy). 


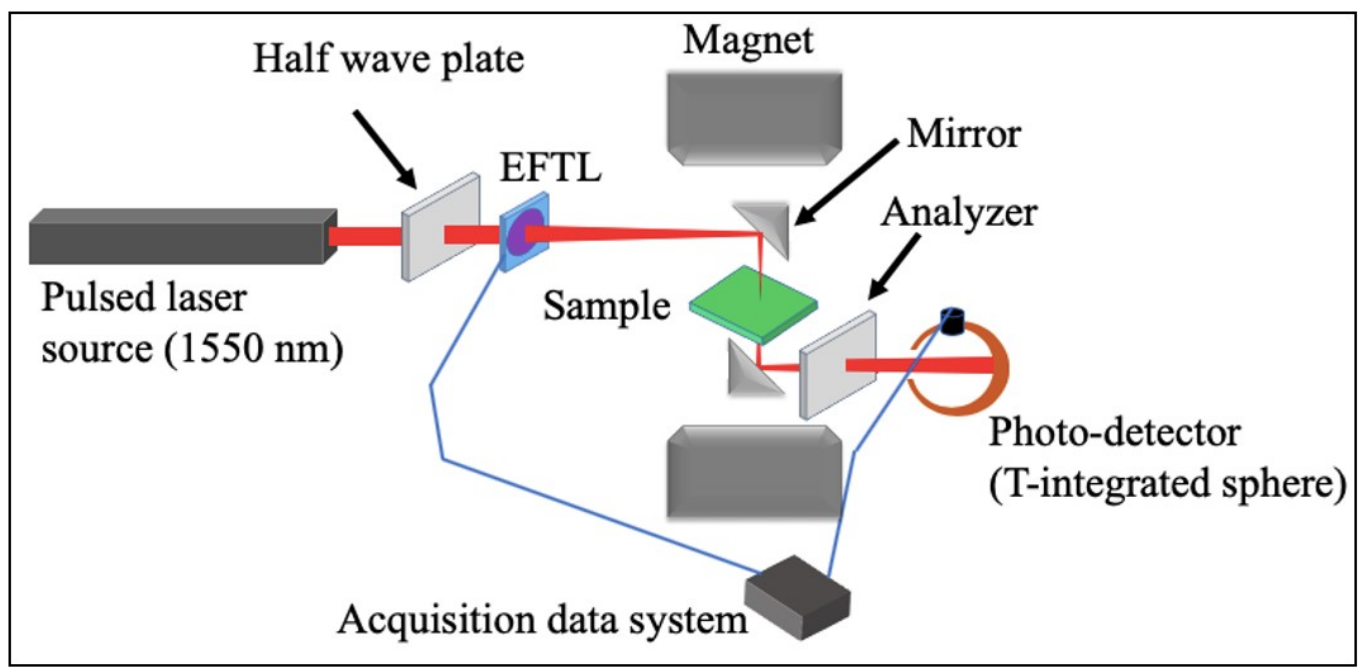

(a)

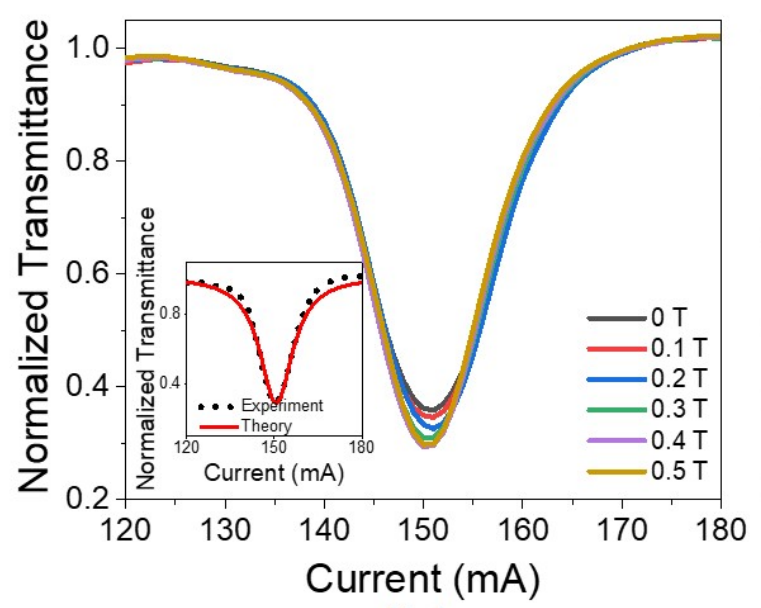

(b)

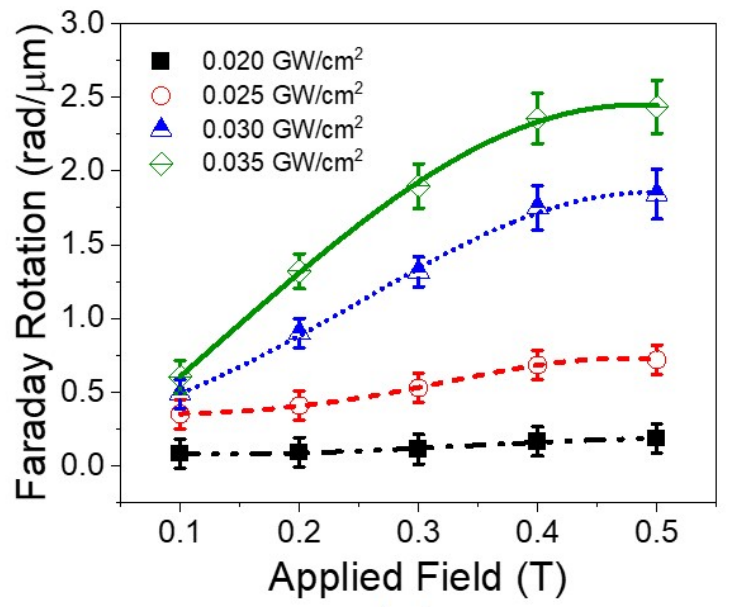

(c)

Figure 4. (a) Schematic diagram for experimental setup of the Nonlinear-polarization rotation F-scan method, (b) normalized transmission at different B-field, inset shows the normalized transmittance for $0.5 \mathrm{~T}$ and the theoretical fitting using equation 9, and the experimental value found for $\chi_{i}^{3}$, (c) Nonlinear polarization rotation with respect to magnetic field at different laser intensities. The solid and dotted lines are the fitted curves using equation 10

The equation 8 contains information about the linear and nonlinear Faraday effects. As we didn't observe any linear Faraday rotation behavior, the observed rotation at higher intensities is due to pure photo-induced Faraday effect. In this case the above expression can be written as

$$
T(f)=\left\{1-\beta I_{o}\left(f+f_{s}\right) L_{e f f}\right\}\left[1-\frac{\chi_{i}^{4}}{2 \chi_{i}^{3}} H \ln \left\{1+\beta I_{o}\left(f+f_{s}\right) L_{e f f}\right\}\right]
$$


with

$$
\Delta \Theta=\frac{\chi_{i}^{4}}{2 \chi_{i}^{3}} H \ln \left\{1+\beta I_{o}\left(f+f_{s}\right) L_{e f f}\right\}
$$

Fig. 4(b) (in inset) and (c) are the experimental transmittance at $0.5 \mathrm{~T}$ and photo-induced (nonlinear) Faraday rotation at different intensities along with fitted data using equation 9 and equation 10 respectively. For fit, first, we calculated the third-order nonlinear susceptibility in terms of the two photon absorption coefficient $(\beta)$ which was obtained to be $\chi_{i}^{3}=\beta \frac{n^{2} c \lambda}{8 \pi^{3}}=9.4 \times 10^{-8}$ esu. Then, $\chi_{i}^{4}$ was obtained by fitting equation 9 at $0.5 \mathrm{~T}$. We found the value for the nonlinear fourth order susceptibility to be $\chi_{i}^{4}=6.5 \times 10^{-12}$ esu. The value obtained for $\chi_{i}^{4}$ is six orders of magnitude larger than in dilute semiconductors [25].

\section{DISCUSSION}

The nonlinear optical processes in metallic nanostructures are generally governed by various excitations like multiphoton absorption, thermal scattering, interband and intraband transitions and geometry [46-48] . In our experiment, we ruled out the possibility of thermal scattering, by using a very low energy pulse of $0.4 \mathrm{~nJ} /$ pulse, with an average power of $20 \mathrm{~mW}$ on the sample, along with a repetition rate of $50 \mathrm{MHz}$, which put the system in a relatively low intensity regimen. Also the excitation wavelength of $1550 \mathrm{~nm}$ was far away from the plasmon resonance and therefore, low linear absorption and consequently low heating of the nanostructures is expected. Additionally, the enhancement of two photon absorption (by four times) of Fe-Ag nanoparticles over that of pure Ag nanoparticles in off-resonant wavelength was attributed to the interband and intraband transition of Fe-metals which subsequently reduced the absorption saturation effect of Fe-Ag system over pure Ag [49]. Surprisingly, we observed that the Fe-Ag nanopyramidal system has an enhancement of about 10 times that of the Ag system at lower photon energy $(1550 \mathrm{~nm})$. Another important observation of the optical nonlinearity of the $\mathrm{Fe}-\mathrm{Ag}$ system over pure $\mathrm{Ag}$ was large as well as negative value of $n_{2}$ which was not reported in the previous study. It indicates the Fe-Ag system is self-defocusing while the pure Ag system is self-focusing. This is likely due to the difference in local optical response/polarization of $\mathrm{Fe}$ and $\mathrm{Ag}$ nanopyramids with incident laser fields as discussed above. Of particular interest was the same Fe-Ag system with the extraordinarily large value of nonlinear (photo-induced) Faraday rotation and nonlinear magneto-optical susceptibility at the same wavelength. While the EELS results strongly suggest a contribution from plasmon induced activity in the origin of the giant nonlinear properties of this metallic system, other mechanisms may still be important. For instance, one can expect the enhancement in the optical nonlinearity of the $\mathrm{Fe}-\mathrm{Ag}$ system due to evanescent wave intensification at the magnetic/nonmagnetic interface through Schoch effect (magnetoelastic origin) and/or Goos-Hänchen effect (electromagnetic origin) 
[50, 51]. The observation of large optical nonlinearity and strong nonlinear (photo-induced) polarization rotation effect on this system at the same wavelength of $1550 \mathrm{~nm}$ is very unique and clearly suggests an exciting system for further exploration.

\section{CONCLUSION}

To summarize, we investigated the nonlinear optical and magneto-optical properties of bi-metallic nanopyramidal systems in telecommunication wavelength. We found that the giant value of nonlinear optical and magneto-optical coefficients are due to two photon absorption phenomena which is further enhanced due to plasmon induced synergistic effect between two metallic systems. We further found that the partially overlapped Fe-Ag system exhibits a negative and extraordinarily large value of third order refractive index, two photon absorption coefficient and nonlinear Faraday rotation unlike pure Ag or Fe nanopyramidal system. Moreover, the nonlinear responses in Fe-Ag systems are strongly dependent on the overlapping extent and in turn the plasmon induced dipole strength across the ferromagnetic material. This strongly indicates the giant enhancement of third order nonlinearity in $\mathrm{Fe}-\mathrm{Ag}$ nanopyramidal systems due to the plasmon induced activity in Fe metals like magneto-electric effect which depends on the plasmon induced polarization and magnetization of ferromagnetic material. All these properties in a single nanosystem brings many fold increase in the technological potentials such as for the realization of various miniaturized all-optical, magneto-optical, quantum-optical and integrated devices at telecommunication wavelengths.

\section{METHODS}

\section{Angle-resolved nanosphere lithography}

To fabricate the pyramidal metallic nanostructures from the nanosphere lithography, the polystyrene beads of diameter $500 \mathrm{~nm}$ were used to mask the quartz substrates by the technique used by Prasad et al [34]. The partial overlapping in nanopyramids was achieved by tilting the masked substrates on the sample holder away to the metallic vapor direction. To achieve $90 \%$ overlapped in between Fe and $\mathrm{Ag}$ sample holder was tilted away by $1^{\circ}$ to vapor direction, while to achieve $30 \%$, the sample holder was tilted away by $5^{\circ}$. 


\section{EELS fit}

In order to quantify the plasmon peak position and peak area- Gaussian peaks and Lorentzian peaks were fit to the data. A multiplication of the Lorentzian functions was used to approximate the zero loss peak. A fit of zero loss peak is shown in Fig. 5 .
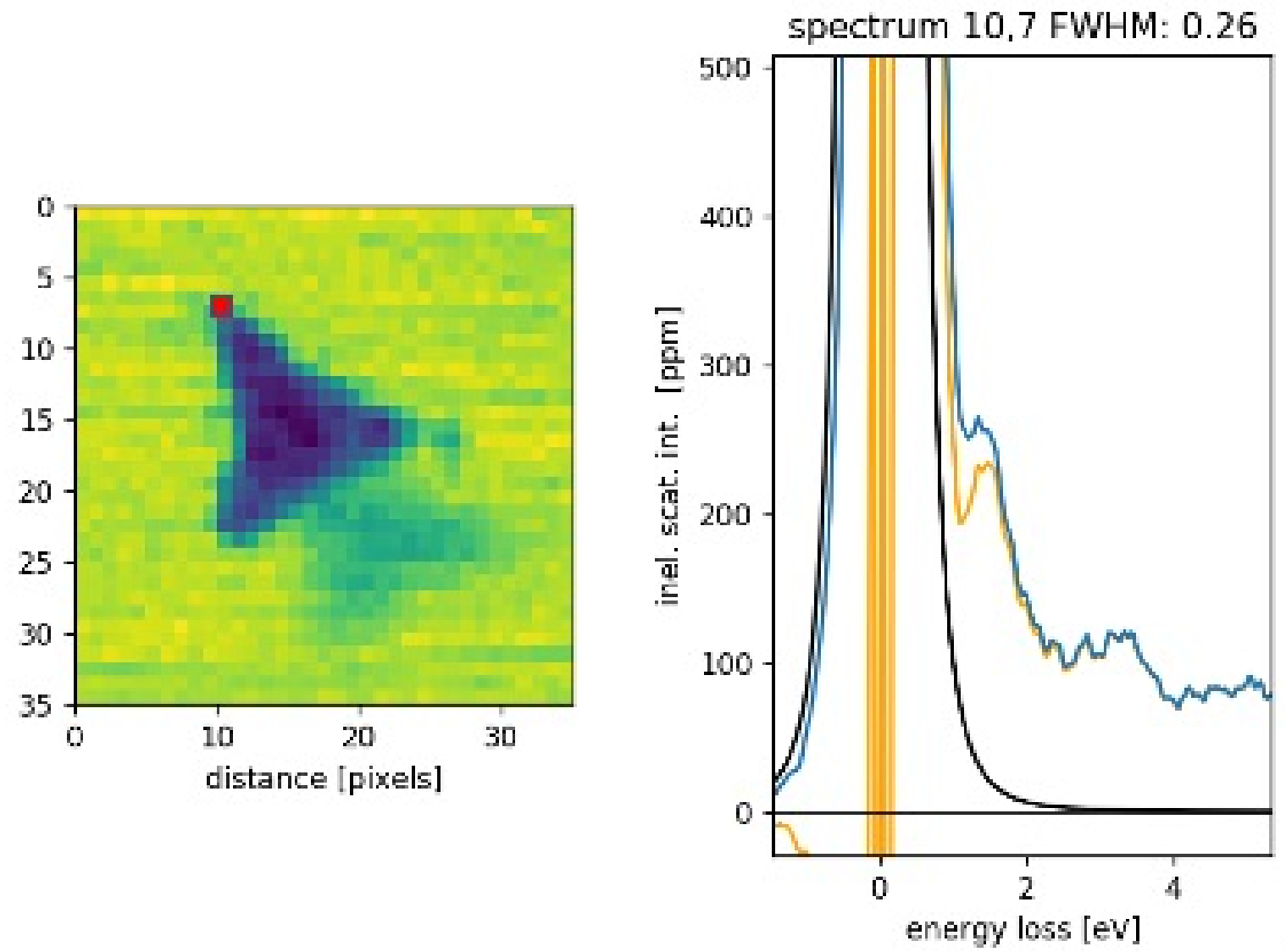

Figure 5. Fit spectra of zero-loss spectrum peak (black solid line) taken from red box.

The summation of gaussian peaks were used to produce noise free models of the electron energy-loss spectra. Inelastic scattering in ppm (parts per million) was calculated using the relation: $\frac{I \times 10^{6}}{I_{0}}$ Where $I$ is the beam current in a pixel from the spectrum and $I_{0}$ is the incident beam current. The Gaussian peak corresponding to the dipole plasmon peak position was used to analyze the area and peak intensity (in ppm) of plasmon loss for corresponding position (A, B and C). A representative of fit data is shown in Fig. 6 . 

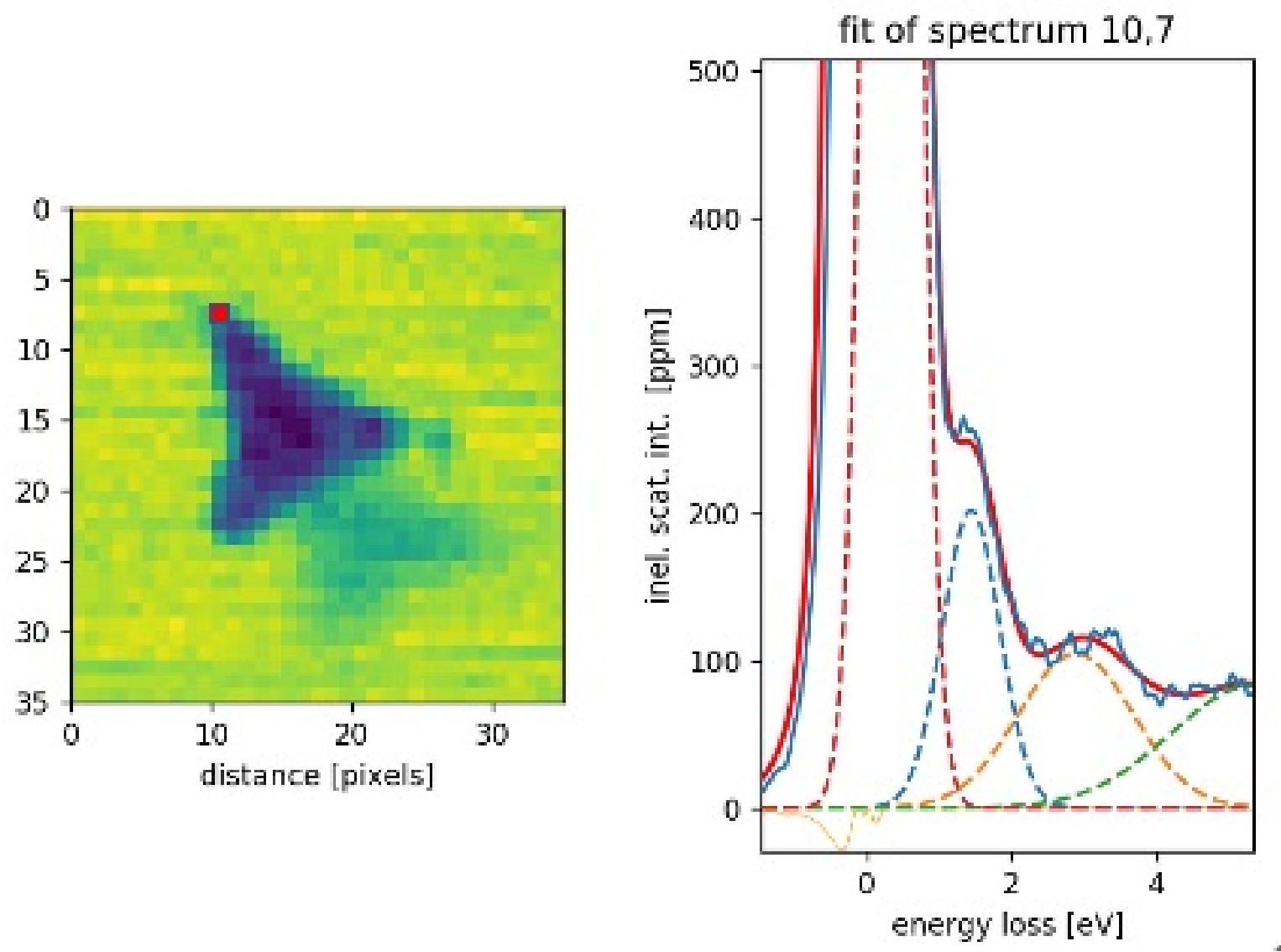

Figure 6. A representative of fitting of plasmon-loss peak for the spectrum data taken from the red box

\section{ACKNOWLEDGMENT}

The authors want to acknowledge support by NSF Grant ECCS-1607874. HG acknowledges financial support from SIUE. GD acknowledges support by U.S. Department of Energy, Office of Science, Basic Energy Sciences, Materials Sciences and Engineering Division

[1] Yi Hua, Kavita Chandra, Duncan Hieu M Dam, Gary P Wiederrecht, and Teri W Odom. Shape-dependent nonlinear optical properties of anisotropic gold nanoparticles. The journal of physical chemistry letters, 6(24):49044908, 2015.

[2] Kai Wang, Hua Long, Ming Fu, Guang Yang, and Peixiang Lu. Size-related third-order optical nonlinearities of au nanoparticle arrays. Optics express, 18(13):13874-13879, 2010.

[3] Fei Zhao, Hao-Ling Sun, Gang Su, and Song Gao. Synthesis and size-dependent magnetic properties of monodisperse eus nanocrystals. small, 2(2):244-248, 2006. 
[4] Chung-Lun Wu, Yung-Hsiang Lin, Sheng-Pin Su, Bo-Ji Huang, Cheng-Ting Tsai, Huai-Yung Wang, Yu-Chieh Chi, Chih-I Wu, and Gong-Ru Lin. Enhancing optical nonlinearity in a nonstoichiometric sin waveguide for cross-wavelength all-optical data processing. Acs Photonics, 2(8):1141-1154, 2015.

[5] Mansoor Sheik-Bahae, Ali A Said, David J Hagan, MJ Soileau, and Eric W Van Stryland. Nonlinear refraction and optical limiting in. Optical engineering, 30(8):1228-1236, 1991.

[6] Richard D Schaller, Justin C Johnson, Kevin R Wilson, Lynn F Lee, Louis H Haber, and Richard J Saykally. Nonlinear chemical imaging nanomicroscopy: from second and third harmonic generation to multiplex (broadbandwidth) sum frequency generation near-field scanning optical microscopy, 2002.

[7] Michael Scalora, Jonathan P Dowling, Charles M Bowden, and Mark J Bloemer. Optical limiting and switching of ultrashort pulses in nonlinear photonic band gap materials. Physical review letters, 73(10):1368, 1994.

[8] T Morioka, S Kawanishi, H Takara, and M Saruwatari. Multiple-output, 100 gbit/s all-optical demultiplexer based on multichannel four-wave mixing pumped by a linearly-chirped square pulse. Electronics Letters, 30(23):1959-1960, 1994.

[9] Dirk Jalas, Nabeel Hakemi, Matteo Cherchi, Mikko Harjanne, Alexander Yu Petrov, and Manfred Eich. Faraday rotation in silicon waveguides. In 2017 IEEE 14th International Conference on Group IV Photonics (GFP), pages 141-142. IEEE, 2017.

[10] R Dekker, A Driessen, T Wahlbrink, C Moormann, J Niehusmann, and M Först. Ultrafast kerr-induced all-optical wavelength conversion in silicon waveguides using $1.55 \mu \mathrm{m}$ femtosecond pulses. Optics express, 14(18):83368346, 2006.

[11] PA Andrekson, NA Olsson, JR Simpson, T Tanbun-Ek, RA Logan, and M Haner. 16 gbit/s all-optical demultiplexing using four-wave mixing. Electronics Letters, 27(11):922-924, 1991.

[12] Rafael Drampyan, Andrew D Greentree, and AV Durrant. Two field nonlinear faraday rotation in rubidium vapor in a doppler-free geometry. Optics communications, 276(2):251-260, 2007.

[13] Florian Wolfgramm, Alessandro Cere, Federica A Beduini, Ana Predojević, Marco Koschorreck, and Morgan W Mitchell. Squeezed-light optical magnetometry. Physical review letters, 105(5):053601, 2010.

[14] M Dinu, Francesco Quochi, and H Garcia. Third-order nonlinearities in silicon at telecom wavelengths. Applied physics letters, 82(18):2954-2956, 2003.

[15] Han Zhang, Stéphane Virally, Qiaoliang Bao, Loh Kian Ping, Serge Massar, Nicolas Godbout, and Pascal Kockaert. Z-scan measurement of the nonlinear refractive index of graphene. Optics letters, 37(11):1856-1858, 2012.

[16] Xiang Qi, Yupeng Zhang, Qingdong Ou, Son Tung Ha, Cheng-Wei Qiu, Han Zhang, Yi-Bing Cheng, Qihua Xiong, and Qiaoliang Bao. Photonics and optoelectronics of $2 \mathrm{~d}$ metal-halide perovskites. Small, 14(31):1800682, 2018.

[17] Manish Chhowalla, Zhongfan Liu, and Hua Zhang. Two-dimensional transition metal dichalcogenide (tmd) nanosheets. Chemical Society Reviews, 44(9):2584-2586, 2015. 
[18] Pengfei Li, Yao Chen, Tieshan Yang, Ziyu Wang, Han Lin, Yanhua Xu, Lei Li, Haoran Mu, Bannur Nanjunda Shivananju, Yupeng Zhang, et al. Two-dimensional ch3nh3pbi3 perovskite nanosheets for ultrafast pulsed fiber lasers. ACS applied materials \& interfaces, 9(14):12759-12765, 2017.

[19] Jingying Liu, Yunzhou Xue, Ziyu Wang, Zai-Quan Xu, Changxi Zheng, Bent Weber, Jingchao Song, Yusheng Wang, Yuerui Lu, Yupeng Zhang, et al. Two-dimensional ch3nh3pbi3 perovskite: Synthesis and optoelectronic application. ACS nano, 10(3):3536-3542, 2016.

[20] Renzhi Ma and Takayoshi Sasaki. Nanosheets of oxides and hydroxides: ultimate 2d charge-bearing functional crystallites. Advanced materials, 22(45):5082-5104, 2010.

[21] Khant Minn, Aleksei Anopchenko, Jingyi Yang, and Ho Wai Howard Lee. Excitation of epsilon-near-zero resonance in ultra-thin indium tin oxide shell embedded nanostructured optical fiber. Scientific reports, 8(1):2342, 2018.

[22] Kostya S Novoselov, D Jiang, F Schedin, TJ Booth, VV Khotkevich, SV Morozov, and Andre K Geim. Twodimensional atomic crystals. Proceedings of the National Academy of Sciences, 102(30):10451-10453, 2005.

[23] Yunzhou Xue, Yupeng Zhang, Yan Liu, Hongtao Liu, Jingchao Song, Joice Sophia, Jingying Liu, Zaiquan Xu, Qingyang Xu, Ziyu Wang, et al. Scalable production of a few-layer mos2/ws2 vertical heterojunction array and its application for photodetectors. Acs Nano, 10(1):573-580, 2015.

[24] RW Cooper, WA Crossley, JL Page, and RF Pearson. Faraday rotation in yig and tbig. Journal of Applied Physics, 39(2):565-567, 1968.

[25] J Frey, R Frey, C Flytzanis, and R Triboulet. Observation of nonlinear faraday processes in cdmnte diluted magnetic semiconductors. Optics communications, 84(1-2):76-80, 1991.

[26] LM Barkov, DA Melik-Pashayev, and MS Zolotorev. Nonlinear faraday rotation in samarium vapor. Optics communications, 70(6):467-472, 1989.

[27] Vasily V Temnov, Gaspar Armelles, Ulrike Woggon, Dmitry Guzatov, Alfonso Cebollada, Antonio GarciaMartin, Jose-Miguel Garcia-Martin, Tim Thomay, Alfred Leitenstorfer, and Rudolf Bratschitsch. Active magneto-plasmonics in hybrid metal-ferromagnet structures. Nature Photonics, 4(2):107, 2010.

[28] Jessie Yao Chin, Tobias Steinle, Thomas Wehlus, Daniel Dregely, Thomas Weiss, Vladimir I Belotelov, Bernd Stritzker, and Harald Giessen. Nonreciprocal plasmonics enables giant enhancement of thin-film faraday rotation. Nature communications, 4:1599, 2013.

[29] Wei Zheng, Xiao Liu, Aubrey T Hanbicki, Berend T Jonker, and Gunter Lüpke. Nonlinear magneto-plasmonics. Optical Materials Express, 5(11):2597-2607, 2015.

[30] DC Marinica, AK Kazansky, Peter Nordlander, Javier Aizpurua, and Andrei G Borisov. Quantum plasmonics: nonlinear effects in the field enhancement of a plasmonic nanoparticle dimer. Nano letters, 12(3):1333-1339, 2012.

[31] Krishna Prasad Koirala, Venkatanarayana Prasad Sandireddy, Hernando Garcia, Gerd Duscher, and Ramki Kalyanaraman. Nanosecond switchable localized surface plasmonsthrough resettable contact angle behavior in silvernanoparticles. Nanotechnology, 2020. 
[32] Ritesh Sachan, S Yadavali, N Shirato, H Krishna, V Ramos, Gerd Duscher, Stephen J Pennycook, AK Gangopadhyay, H Garcia, and R Kalyanaraman. Self-organized bimetallic ag-co nanoparticles with tunable localized surface plasmons showing high environmental stability and sensitivity. Nanotechnology, 23(27):275604, 2012.

[33] Ritesh Sachan, Abhinav Malasi, Jingxuan Ge, Sagar Yadavali, Hare Krishna, Anup Gangopadhyay, Hernando Garcia, Gerd Duscher, and Ramki Kalyanaraman. Ferroplasmons: Intense localized surface plasmons in metalferromagnetic nanoparticles. ACS nano, 8(10):9790-9798, 2014.

[34] Venkatanarayana P Sandireddy, Krishna P Koirala, Humaira Taz, and Ramki Kalyanaraman. Thermal and plasmonic stabilization of silver nanostructures using a bilayer anchoring technique. ACS applied materials \& interfaces, 10(39):33630-33639, 2018.

[35] Prashant K Jain, Yanhong Xiao, Ronald Walsworth, and Adam E Cohen. Surface plasmon resonance enhanced magneto-optics (supremo): Faraday rotation enhancement in gold-coated iron oxide nanocrystals. Nano letters, 9(4):1644-1650, 2009.

[36] Edgar Rueda, Juan H Serna, Abdullatif Hamad, and Hernando Garcia. Two-photon absorption coefficient determination using the differential f-scan technique. Optics \& Laser Technology, 119:105584, 2019.

[37] Juan Serna, Julián Henao, Edgar Rueda, Abdullatif Hamad, and Hernando Garcia. Reflection technique for determination of nonlinear-refractive index of thin-film semiconductors using an electrically focus-tunable lens. In Frontiers in Optics, pages JW4A-12. Optical Society of America, 2019.

[38] H Garcia, J Serna, and E Rueda. Bulk znse and cds two-photon absorption measurement with an f-scan nonlinear absorption spectrometer. OSA Continuит, 3(3):498-504, 2020.

[39] Minjoung Kyoung and Minyung Lee. Nonlinear absorption and refractive index measurements of silver nanorods by the z-scan technique. Optics communications, 171(1-3):145-148, 1999.

[40] Carlos Torres-Torres, Néstor Peréa-López, Jorge Alejandro Reyes-Esqueda, Luis Rodríguez-Fernández, Alejandro Crespo-Sosa, Juan Carlos Cheang-Wong, and Alicia Oliver. Ablation and optical third-order nonlinearities in ag nanoparticles. International journal of nanomedicine, 5:925, 2010.

[41] Baolong Yu, Congshan Zhu, Fuxi Gan, Xiaochum Wu, Guilan Zhang, Guoqing Tang, and Wenju Chen. Optical nonlinearities of fe2o3 nanoparticles investigated by z-scan technique. Optical Materials, 8(4):249-254, 1997.

[42] Ray F Egerton. Electron energy-loss spectroscopy in the electron microscope. Springer Science \& Business Media, 2011.

[43] Chol-Song Ri, Song-Jin Im, Ji-Song Pae, Kum-Song Ho, Yong-Ha Han, and Joachim Herrmann. Nanoscale magnetization and third-order nonlinearity by the plasmon-induced inverse faraday effect in graphene-covered semiconductors. Physical Review B, 100(15):155404, 2019.

[44] Y Tokura. Multiferroics toward strong coupling between magnetization and polarization in a solid. Journal of Magnetism and Magnetic Materials, 310(2):1145-1150, 2007.

[45] J Frey, Robert Frey, Christopher Flytzanis, and R Triboulet. Theoretical and experimental investigation of nonlinear faraday processes in diluted magnetic semiconductors. JOSA B, 9(1):132-142, 1992. 
[46] Alexey V Krasavin, Pavel Ginzburg, and Anatoly V Zayats. Free-electron optical nonlinearities in plasmonic nanostructures: A review of the hydrodynamic description. Laser \& Photonics Reviews, 12(1):1700082, 2018.

[47] Akio Kawasaki, Boris Braverman, Edwin Pedrozo-Peñafiel, Chi Shu, Simone Colombo, Zeyang Li, Özge Özel, Wenlan Chen, Leonardo Salvi, André Heinz, et al. Geometrically asymmetric optical cavity for strong atomphoton coupling. Physical Review A, 99(1):013437, 2019.

[48] Yuri Kivshar. Engineered metaoptics forge new nonlinear devices. LASER FOCUS WORLD, 55(3):39-41, 2019.

[49] Kishore Sridharan, Tamio Endo, Sang-Geun Cho, Jongryoul Kim, Tae Joo Park, and Reji Philip. Single step synthesis and optical limiting properties of ni-ag and fe-ag bimetallic nanoparticles. Optical Materials, 35(5):860867, 2013.

[50] OV Prikhodko, OS Sukhorukova, SV Tarasenko, and VG Shavrov. Evanescent wave intensification in surface spin-wave acoustics and magnetic/nonmagnetic interface electrodynamics. Bulletin of the Russian Academy of Sciences: Physics, 79(6):712-714, 2015.

[51] Henri J Lezec and Tineke Thio. Diffracted evanescent wave model for enhanced and suppressed optical transmission through subwavelength hole arrays. Optics express, 12(16):3629-3651, 2004. 


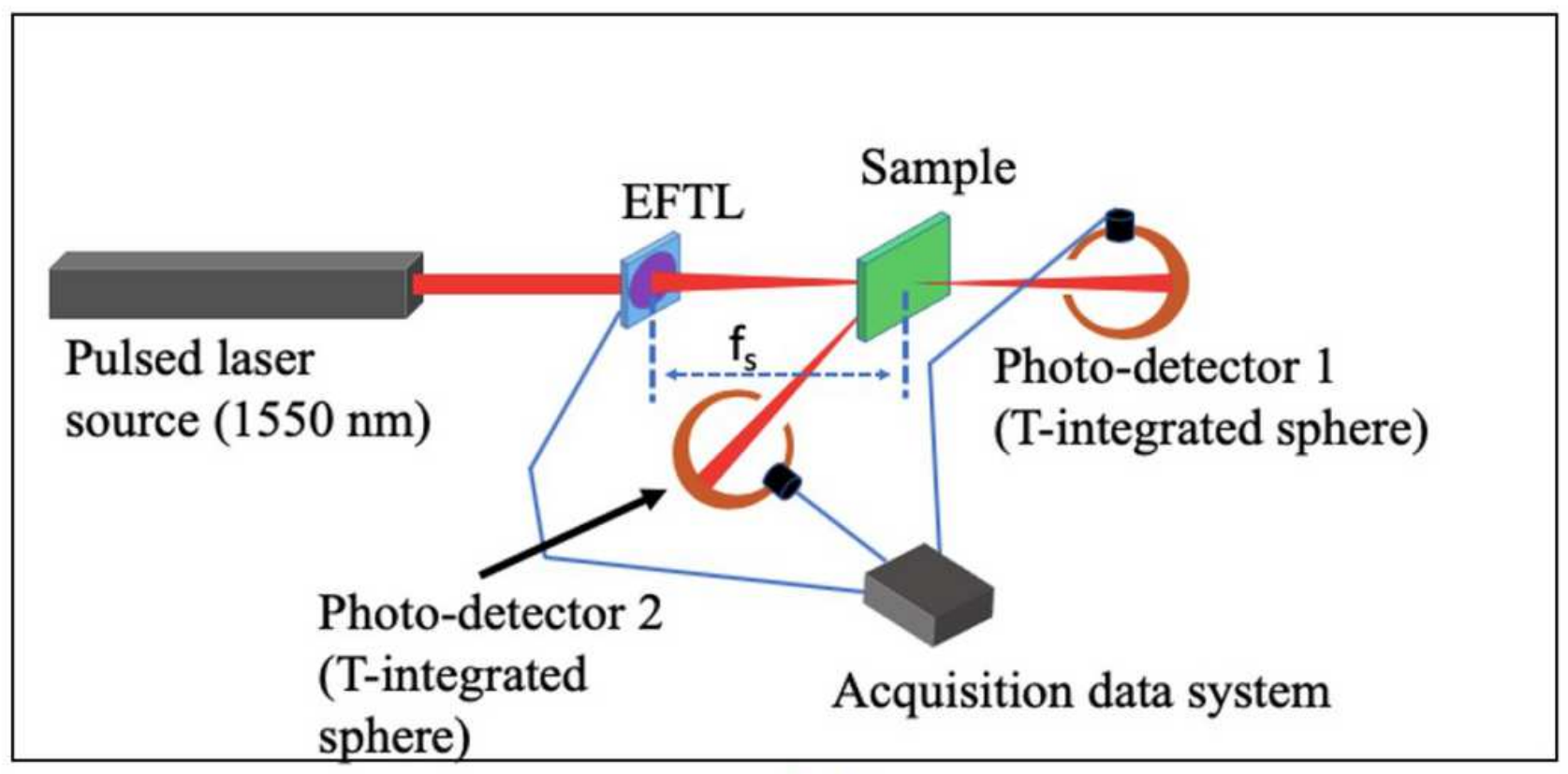

(a)

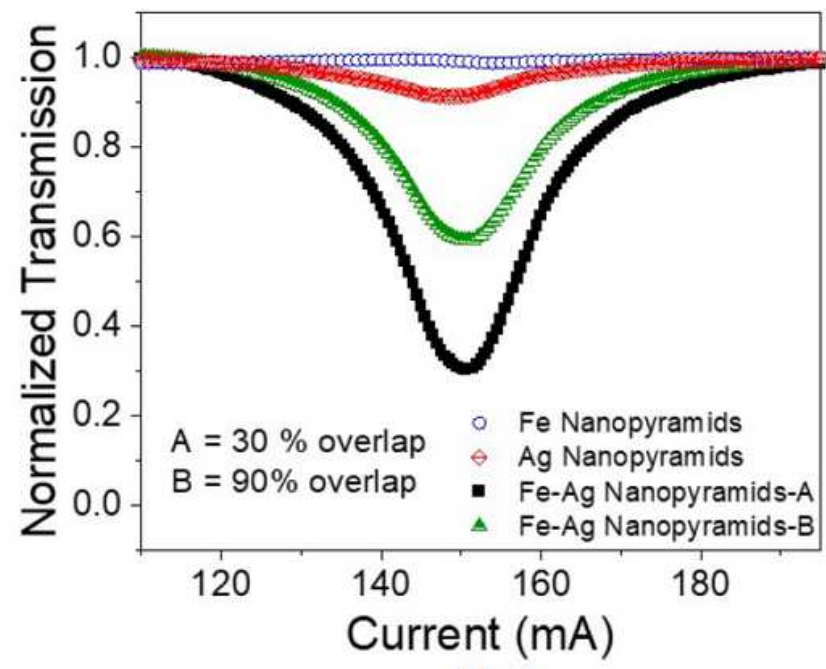

(b)

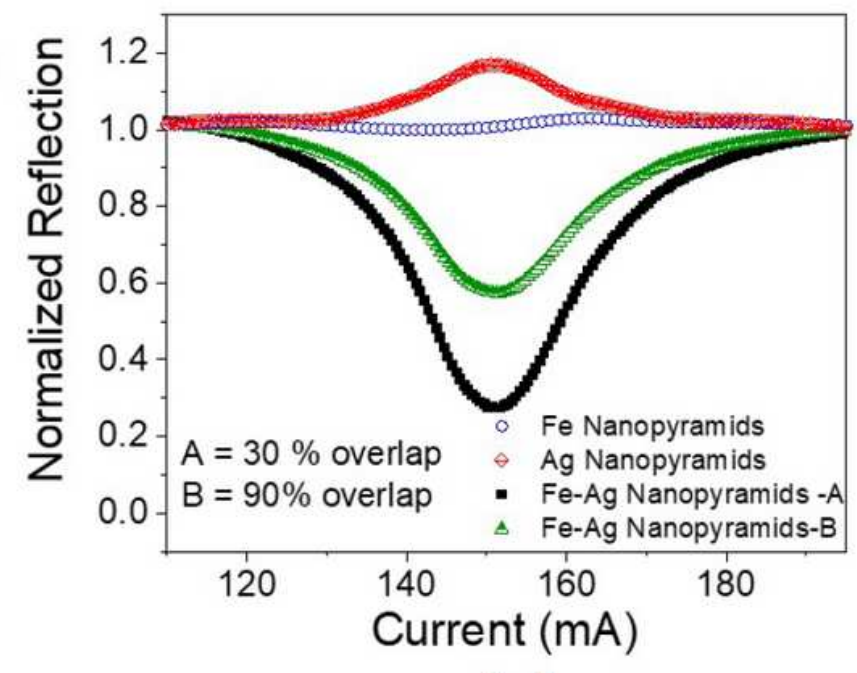

(c)

Figure 1

(a) Schematic diagram for experimental setup of f-scan method (b) normalized transmission and (c) normalized reflection of $\mathrm{Fe}, \mathrm{Ag}$ and Fe-Ag nanopyramidal arrays. 


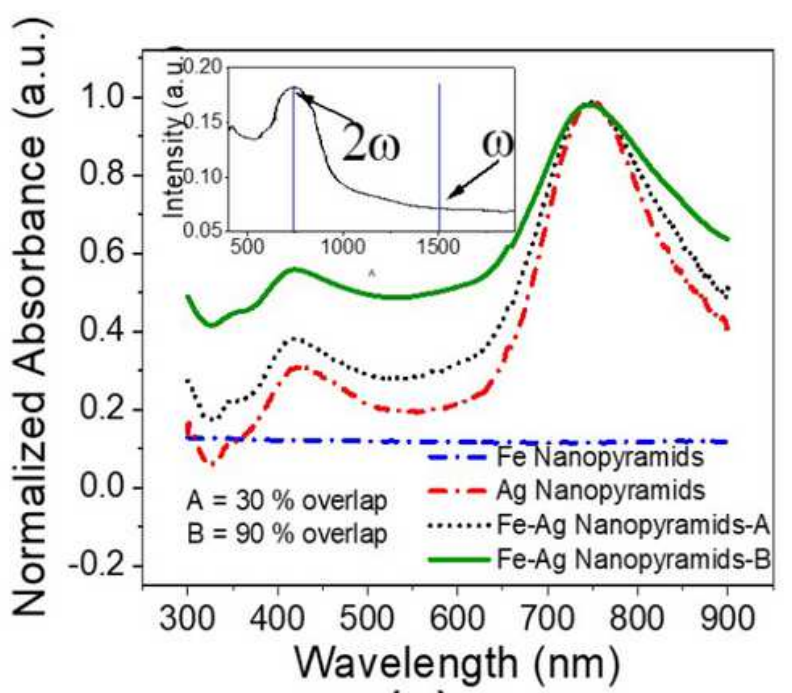

(a)

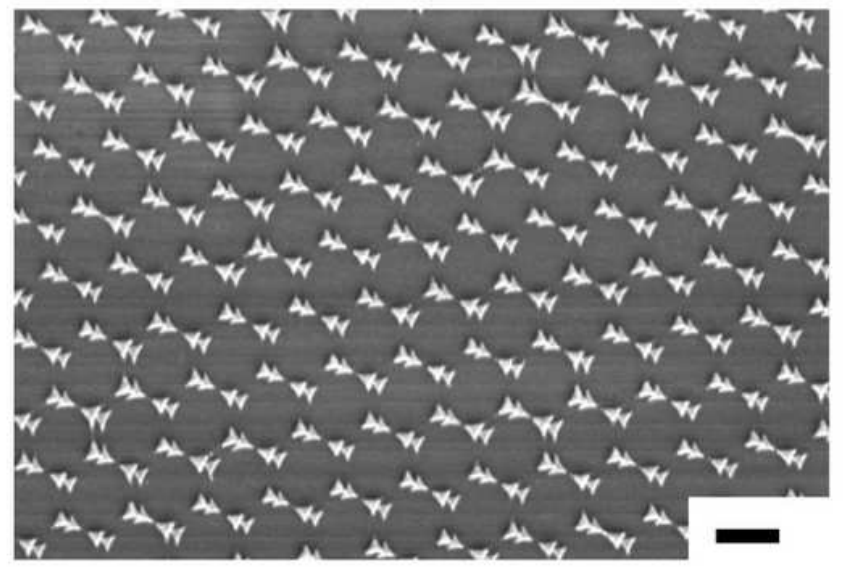

(c)

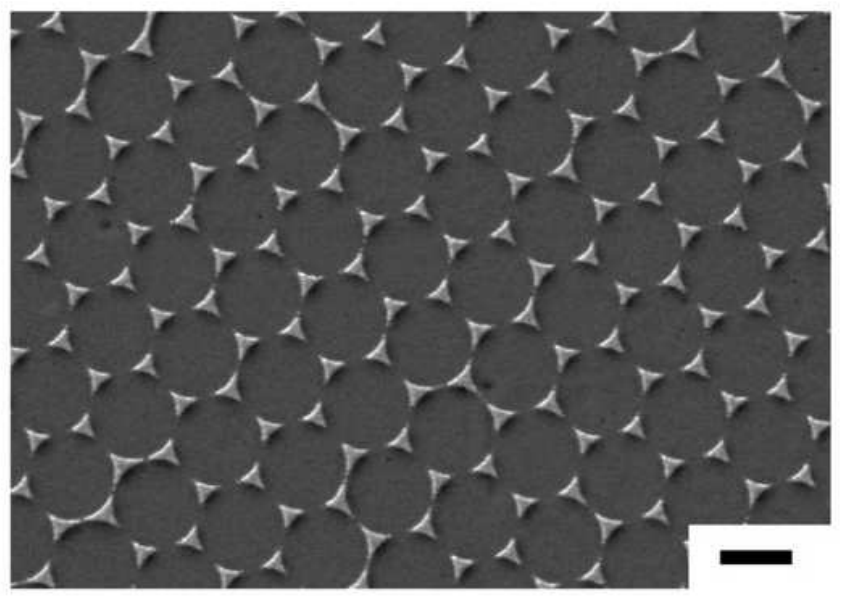

(b)

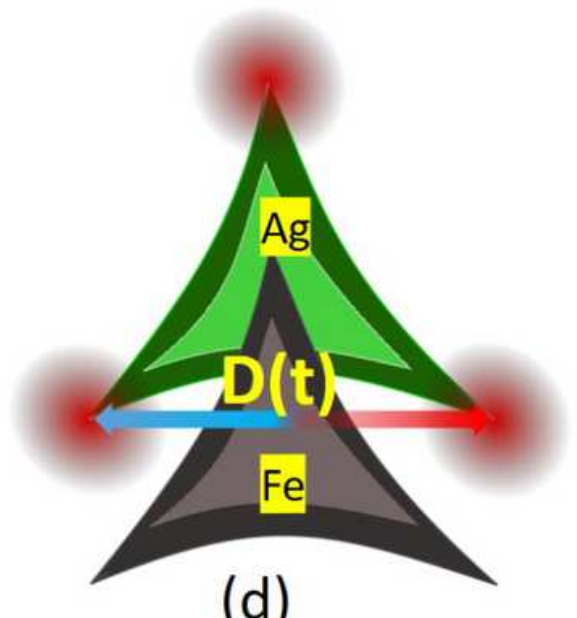

(d)

\section{Figure 2}

(a) Normalized plasmon induced linear absorption spectra of $\mathrm{Fe}, \mathrm{Ag}$ and Fe-Ag (30\% and $90 \%$ overlap) nanopyramidal systems. Inset shows the linear absorption of the Fe-Ag system from 400 to $2000 \mathrm{~nm}$. (b) A representative scanning electron micrograph (SEM) image of pure Ag and (c) Fe-Ag (30\% overlapped system) (the scale bar is $500 \mathrm{~nm}$ ) (d)The schematic of oscillating displacement field $D(t)$ in the Fe pyramid induced by surface plasmon dipoles of a Ag nanopyramid 

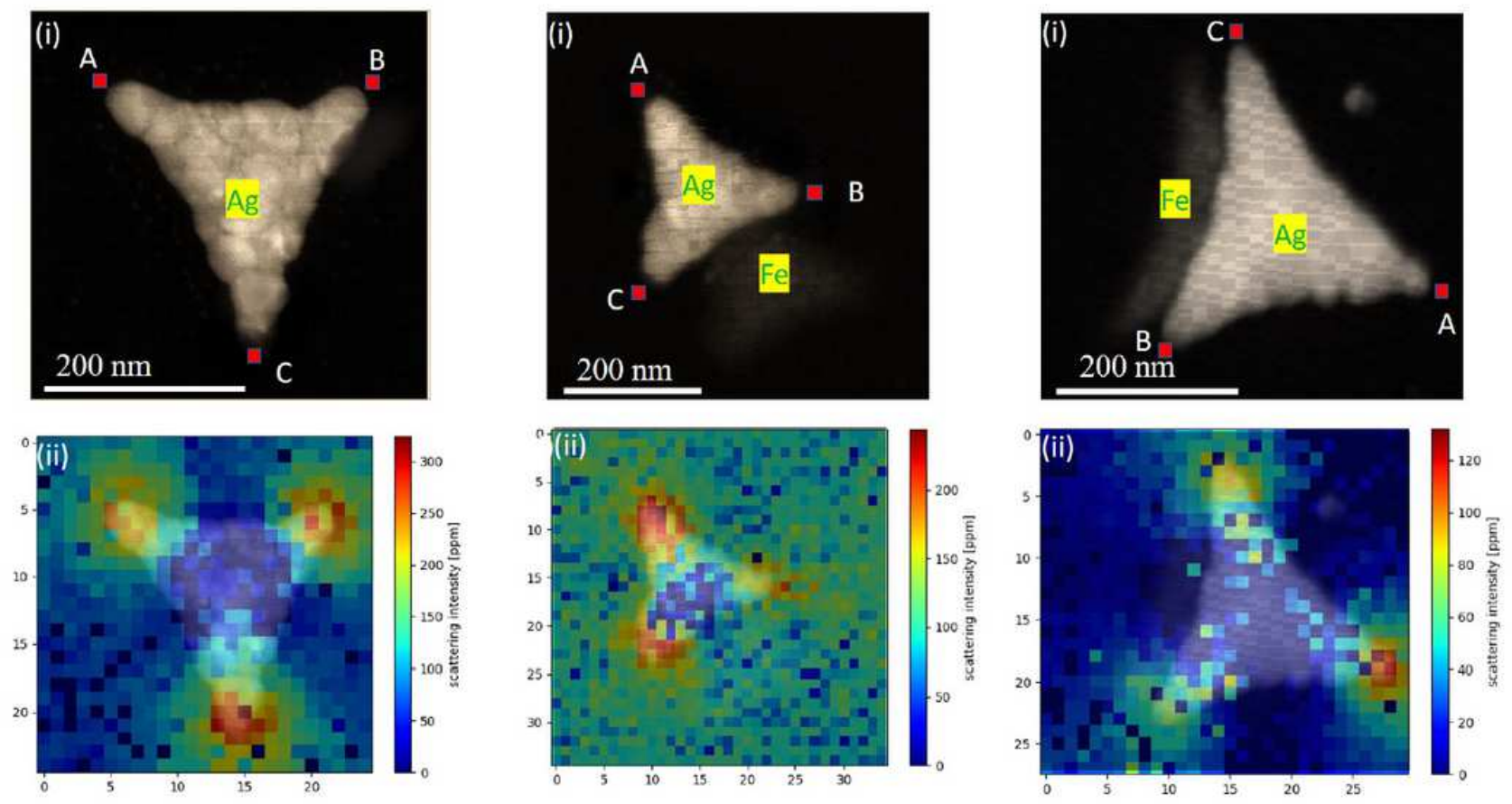

(a)

(b)

(c)

\section{Figure 3}

(i) High-angle annular dark-field images and (ii) EELS intensity mapping of (a) pure Ag, (b) Fe-Ag (30\% overlapped), (c) Fe-Ag (90\% overlapped) nano pyramids at peak value of $1.60 \pm 0.02 \mathrm{eV}$. 


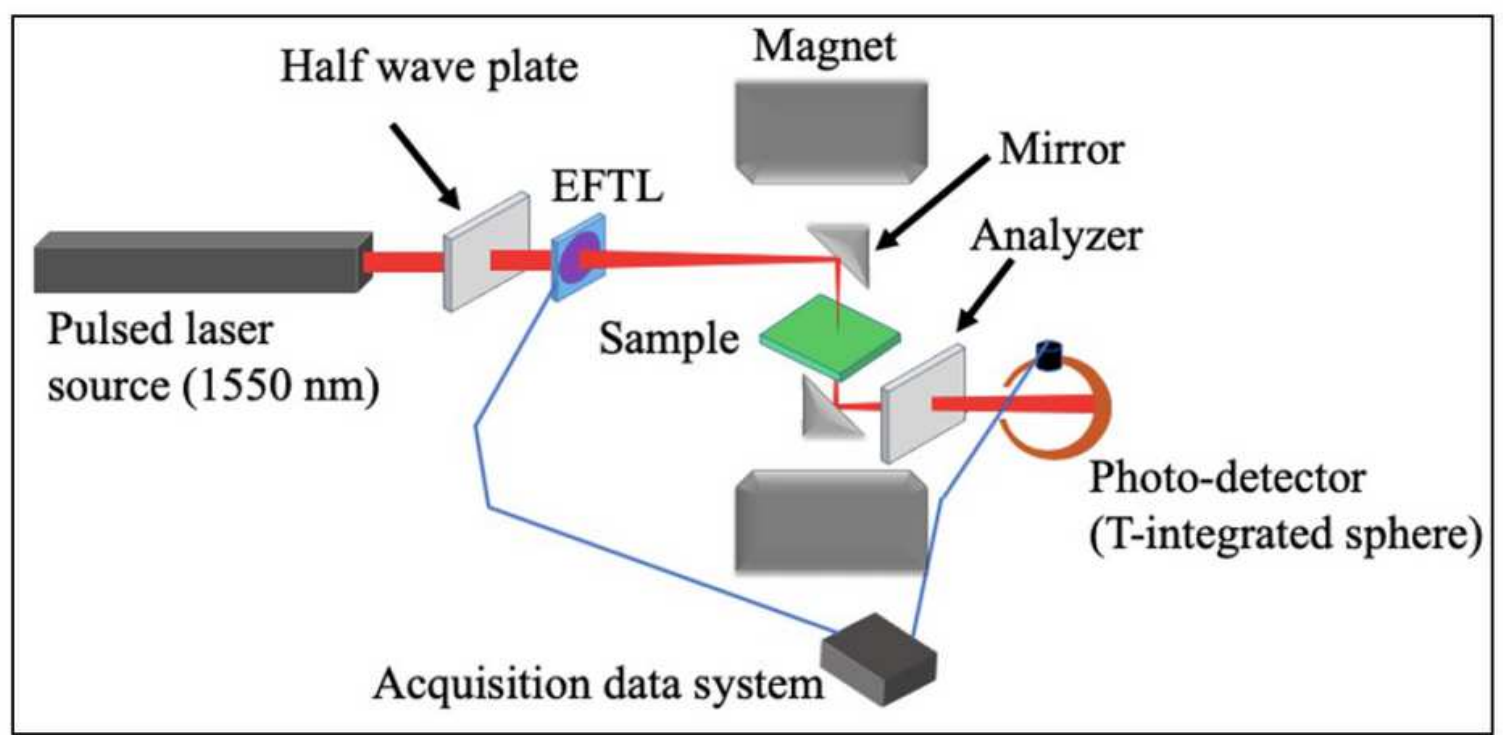

(a)

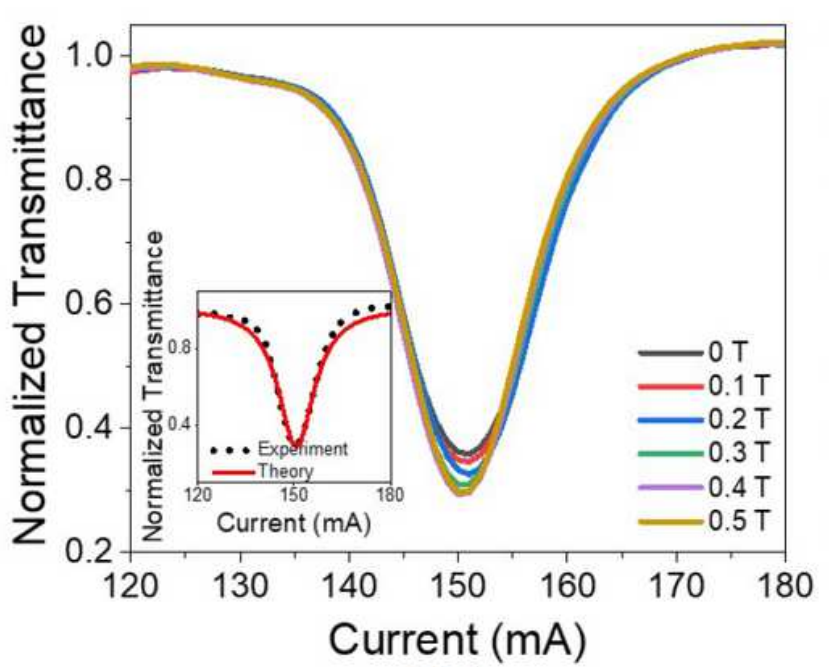

(b)

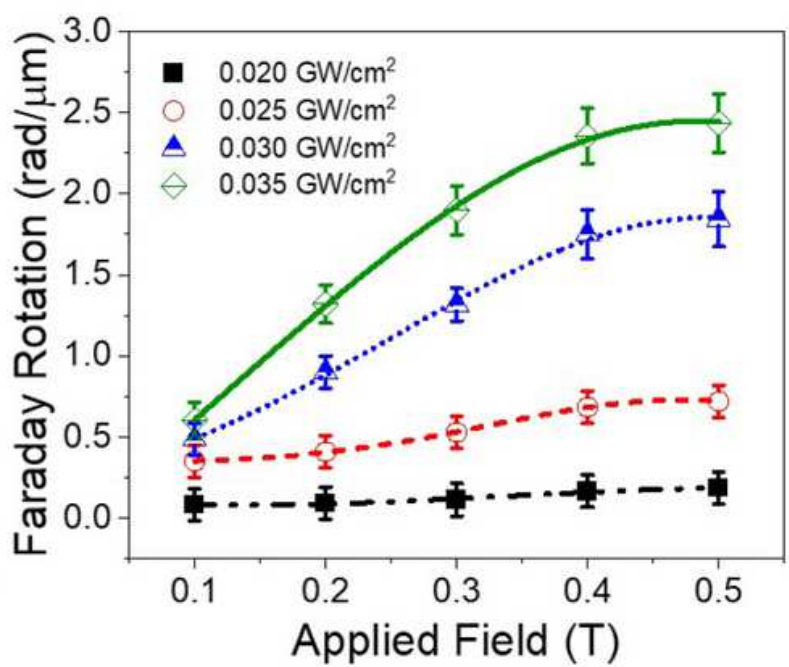

(c)

\section{Figure 4}

(a) Schematic diagram for experimental setup of the Nonlinear-polarization rotation F-scan method, (b) normalized transmission at different B-field, inset shows the normalized transmittance for $0.5 \mathrm{~T}$ and the theoretical fitting using equation 9 , and the experimental value found for $\chi 3 \mathrm{i}$, (c) Nonlinear polarization rotation with respect to magnetic field at different laser intensities. The solid and dotted lines are the fitted curves using equation 10 

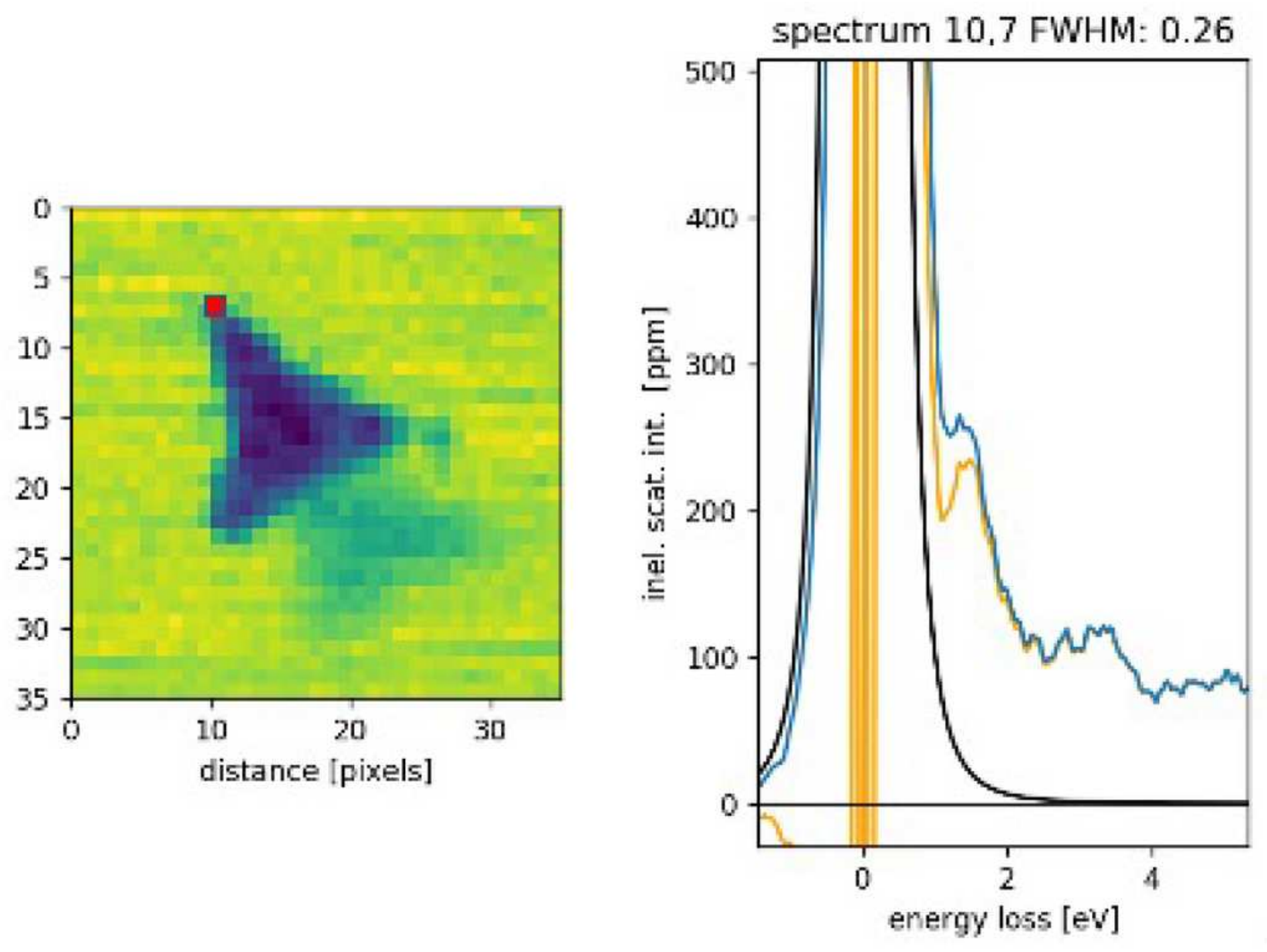

Figure 5

Fit spectra of zero-loss spectrum peak (black solid line) taken from red box. 

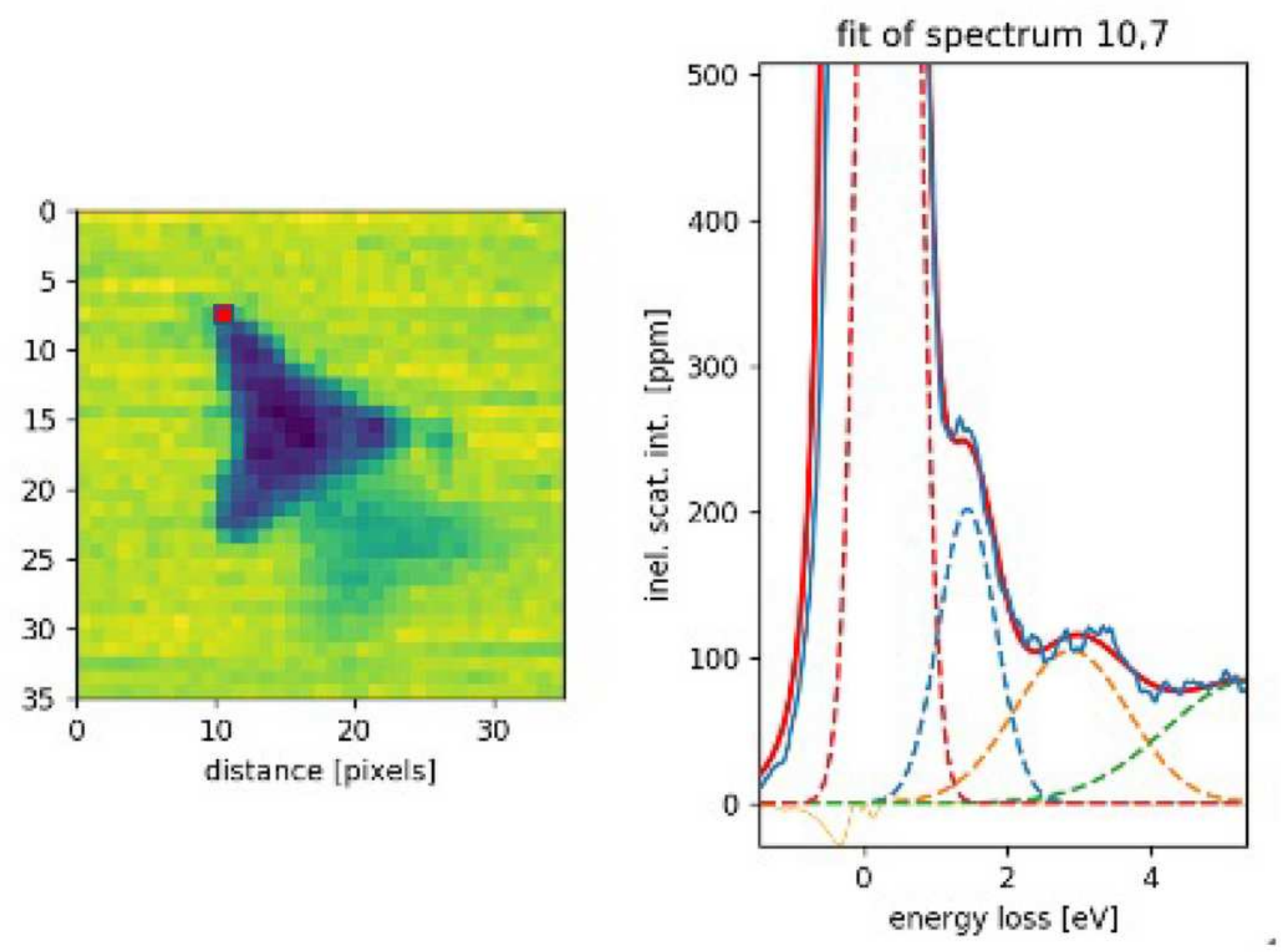

Figure 6

A representative of fitting of plasmon-loss peak for the spectrum data taken from the red box 